\title{
Use of NanoBiT and NanoBRET to monitor fluorescent VEGF-A binding kinetics to VEGFR2/NRP1 heteromeric complexes in living cells
}

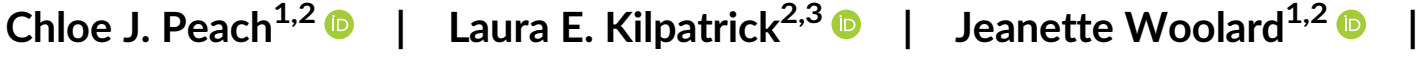 \\ Stephen J. Hill ${ }^{1,2}$ (1)
}

\begin{abstract}
${ }^{1}$ Division of Physiology, Pharmacology and Neuroscience, School of Life Sciences, University of Nottingham, Nottingham, UK

${ }^{2}$ Centre of Membrane Proteins and Receptors, University of Birmingham and University of Nottingham, The Midlands, UK

${ }^{3}$ Division of Bimolecular Sciences and Medicinal Chemistry, Biodiscovery Institute, School of Pharmacy, University of Nottingham, Nottingham, UK
\end{abstract}

\section{Correspondence}

Laura E. Kilpatrick, Division of Bimolecular Sciences and Medicinal Chemistry, Biodiscovery Institute, School of Pharmacy, University of Nottingham, Nottingham NG7 2RD, UK.

Email: laura.kilpatrick@nottingham.ac.uk

Jeanette Woolard and Stephen J. Hill, Division of Physiology, Pharmacology and Neuroscience, School of Life Sciences, University of Nottingham, Nottingham NG7 2UH, UK.

Email: jeanette.woolard@nottingham.ac.uk; stephen.hill@nottingham.ac.uk

Funding information

Biotechnology and Biological Sciences Research Council, Grant/Award Number: BB/ L019418/1; British Pharmacological Society, Grant/Award Number: AJ Clark Studentship for Chloe Peach; Medical Research Council, Grant/Award Number: MR/N020081/1;

University of Nottingham
Background and Purpose: VEGF-A is a key mediator of angiogenesis, primarily signalling via VEGF receptor 2 (VEGFR2). Endothelial cells also express the co-receptor neuropilin-1 (NRP1) that potentiates VEGF-A/VEGFR2 signalling. VEGFR2 and NRP1 had distinct real-time ligand binding kinetics when monitored using BRET. We previously characterised fluorescent VEGF-A isoforms tagged at a single site with tetramethylrhodamine (TMR). Here, we explored differences between VEGF-A isoforms in living cells that co-expressed both receptors.

Experimental Approach: Receptor localisation was monitored in HEK293T cells expressing both VEGFR2 and NRP1 using membrane-impermeant HaloTag and SnapTag technologies. To isolate ligand binding pharmacology at a defined VEGFR2/ NRP1 complex, we developed an assay using NanoBiT complementation technology whereby heteromerisation is required for luminescence emissions. Binding affinities and kinetics of VEGFR2-selective VEGF ${ }_{165}$ b-TMR and non-selective VEGF ${ }_{165}$ a-TMR were monitored using BRET from this defined complex.

Key Results: Cell surface VEGFR2 and NRP1 were co-localised and formed a constitutive heteromeric complex. Despite being selective for VEGFR2, VEGF ${ }_{165} \mathrm{~b}$-TMR had a distinct kinetic ligand binding profile at the complex that largely remained elevated in cells over $90 \mathrm{~min}$. VEGF 165 a-TMR bound to the VEGFR2/NRP1 complex with kinetics comparable to those of VEGFR2 alone. Using a binding-dead mutant of NRP1 did not affect the binding kinetics or affinity of VEGF 165 a-TMR.

Conclusion and Implications: This NanoBiT approach enabled real-time ligand binding to be quantified in living cells at $37^{\circ} \mathrm{C}$ from a specified complex between a receptor TK and its co-receptor for the first time.

\section{KEYWORDS}

BRET, co-receptor, growth factor, kinetics, NanoBiT, receptor tyrosine kinase 


\section{1 | INTRODUCTION}

Angiogenesis involves the growth of new blood vessels from existing vascular networks (Carmeliet, 2005). This important physiological process can also be dysregulated in numerous pathologies, such as in tumour development (Chung \& Ferrara, 2011). VEGF-A is a key mediator of angiogenesis that primarily signals via its cognate receptor tyrosine kinase (RTK), the VEGF receptor 2 (VEGFR2) (Peach, Mignone, et al., 2018; Simons et al., 2016). VEGF-A binds across immunoglobulin-like domains 2 and 3 of VEGFR2 (Leppanen et al., 2010; Ruch et al., 2007). Agonist binding results in conformational changes throughout the VEGFR2 dimer that lead to auto- and trans-phosphorylation of key intracellular tyrosine residues. This triggers numerous signalling cascades that ultimately initiate endothelial cell proliferation, migration, and survival, as well as increased vascular permeability (Koch et al., 2011).

VEGFR2 is subject to complex trafficking via clathrin-dependent and clathrin-independent endocytosis (Basagiannis \& Christoforidis, 2016; Basagiannis et al., 2016; Ewan et al., 2006). It internalises in both the presence and absence of VEGF-A (Ewan et al., 2006; Jopling et al., 2009, 2011). VEGF-A can also bind to the VEGFR2 co-receptor neuropilin-1 (NRP1), a type 1 transmembrane glycoprotein (Soker et al., 1998, 2002). VEGFR2 signalling is up-regulated by NRP1 (Djordjevic \& Driscoll, 2013; Fantin et al., 2011; Gelfand et al., 2014). Endothelial cells express both VEGFR2 and NRP1 (Soker et al., 1998; Witmer et al., 2002). NRP1 is also overexpressed in numerous tumour subtypes (Goel \& Mercurio, 2013; Jubb et al., 2012; Lee et al., 2014) and immune cells in the tumour micro-environment (Roy et al., 2017). Aberrant VEGFR2 signalling in tumour angiogenesis is therefore up-regulated by NRP1 but existing anti-tumour drugs only target VEGF-A/VEGFR2 signalling. VEGF-A interacts with VEGFR2 via residues encoded at the $\mathrm{N}$-terminus of VEGF-A (Brozzo et al., 2011; Leppanen et al., 2010), while the $C$-terminus can interact with NRP1 (Mamluk et al., 2002; Parker et al., 2012; Vander Kooi et al., 2007).

VEGF-A is an anti-parallel, disulphide-linked homodimer. Alternative splicing of VEGF-A mRNA leads to a number of distinct VEGF-A isoforms (Peach, Mignone, et al., 2018; Woolard et al., 2009). VEGF-A isoforms have different signalling properties in physiological systems with distinct expression profiles in health and disease (Vempati et al., 2014). VEGF-A isoforms differ in length, such as pro-angiogenic VEGF $_{165}$ a or the shorter VEGF $_{121}$ a isoform. A major site of splicing occurs at exon 8, where proximal splicing results in $\mathrm{VEGF}_{x x x} \mathrm{a}$ isoforms that contain exon 8a-encoded residues (CDKPRR) and VEGF $_{x x x} b$ isoforms that instead contain exon 8b-encoded residues (SLTKDD). While VEGF 165 a stimulates angiogenesis as a full agonist, VEGF V $_{165} \mathrm{~b}$ is a partial agonist with reported anti-angiogenic activity in vivo (Cébe Suarez et al., 2006; Eswarappa et al., 2014; Woolard et al., 2004). The b1 domain of NRP1 can interact with VEGF 165 a via an arginine residue encoded by exon 8a (Mamluk et al., 2002; Parker et al., 2012; Vander Kooi et al., 2007). In contrast, "anti-angiogenic" VEGF ${ }_{165}$ b isoforms are unable to interact with NRP1 (Cébe Suarez et al., 2006; Delcombel et al., 2013; Kawamura et al., 2008).

\section{What is already known}

- Endothelial cells and tumour cells express both VEGFR2 and its co-receptor NRP1.

- VEGFR2 and NRP1 have distinct ligand binding kinetics and receptor localisation when expressed alone.

\section{What this study adds}

- Real-time assay quantifying fluorescent VEGF-A binding at defined heteromeric complexes in living cells at $37^{\circ} \mathrm{C}$.

\section{What is the clinical significance}

- Aberrant VEGFR2 signalling in cancer is up-regulated by NRP1; however, existing drugs only target VEGF-A/ VEGFR2.

- NRP1 is a promising target in oncology due to its high expression localised to tumours.

Fluorescence-based technologies have been used to advance our pharmacological understanding of GPCRs, RTKs, and other classes of membrane protein (Stoddart et al., 2017). For example, bioluminescence resonance energy transfer (BRET) is a proximity-based assay that can quantify real-time binding at $37^{\circ} \mathrm{C}$ in living cells (Stoddart et al., 2015). A receptor is tagged at the $\mathrm{N}$-terminus with a 19-kDa NanoLuciferase (NanoLuc) such that NanoLuc emits luminescence upon oxidation of the furimazine substrate. This can excite a nearby fluorophore in close proximity $(<10 \mathrm{~nm})$, such as a compatible fluorescent ligand bound at the receptor's orthosteric site. We previously developed fluorescent VEGF-A isoforms that were single site labelled with tetramethylrhodamine (TMR), to monitor ligand binding at fulllength VEGFR2 or NRP1 tagged with NanoLuc (Kilpatrick et al., 2017; Peach, Kilpatrick, et al., 2018; Peach et al., 2019). Despite having a similar nanomolar binding affinity, VEGF ${ }_{165}$ a-TMR binding kinetics were significantly faster at NRP1 than VEGFR2 (Peach, Kilpatrick, et al., 2018). VEGFR2 and NRP1 were also subject to distinct subcellular trafficking in the absence or presence of ligand when expressed alone. These techniques were limited to quantifying protein-protein interactions at NanoLuc-tagged VEGFR2 or NRP1 expressed in isolation; however, endothelial cells and tumour cells endogenously express both VEGFR2 and NRP1 in the same cell (Fantin et al., 2013; Koch et al., 2014; Lee-Montiel et al., 2015; Prahst et al., 2008; Whitaker et al., 2001). As these receptors have distinct ligand binding dynamics and subcellular localisation, approaches are required that isolate the pharmacology of VEGF-A ligand binding to distinct complexes involving both VEGFR2 and NRP1.

NanoLuc Binary Technology (NanoBiT) uses a modified NanoLuc split into a large fragment (LgBiT; 156 amino acids) and a small 
11-amino-acid tag (HiBiT or SmBiT; Dixon et al., 2016). Complementation of fragments is required for luminescence emission. Numerous variants were developed of the small tag with different intrinsic affinities for complementation with the LgBiT fragment, including the "higher affinity" HiBiT fragment $\left(K_{d} \sim 0.7 \mathrm{nM}\right)$ and the lower affinity SmBiT fragment $\left(K_{d} \sim 190 \mu \mathrm{M}\right)$. Used in combination with a fluorescent ligand, interactions between the ligand and a particular protein pairing can be monitored using NanoBiT and BRET. Here, we have used this technology to investigate the kinetics of ligand binding of VEGF $_{165}$ a-TMR (Kilpatrick et al., 2017) and VEGF 165 b-TMR (Peach, Kilpatrick, et al., 2018) to oligomeric complexes containing both VEGFR2 and NRP1.

\section{2 | METHODS}

\section{$2.1 \quad$ Cell culture}

HEK293T cells (CCLV Cat\# CCLV-RIE 1018, RRID:CVCL_0063) were maintained at $37^{\circ} \mathrm{C} / 5 \% \mathrm{CO}_{2}$ in DMEM (Sigma-Aldrich, USA) supplemented with $10 \%$ fetal calf serum (FCS; Sigma-Aldrich). For a consistent cell background with functional studies performed using a reporter gene assay, all HEK293T cells also expressed a Firefly luciferase reporter gene (RE-Luc2P) that was inserted downstream of the NFAT promoter. Control experiments confirmed that HEK293T-NFAT-ReLuc2P cells did not emit luminescence in response to furimazine alone that interfered with NanoBiT or NanoBRET assays. Cells were passaged at $70-80 \%$ confluency using PBS (Lonza, Switzerland) and trypsin ( $0.25 \% \mathrm{w} / \mathrm{v}$ in versene; Lonza).

\subsection{Generating constructs}

N-terminal NanoLuc-tagged VEGFR2 (NM_002253) and NRP1 (NM_003873.5) were cloned in a pFN31K vector encoding the secretory IL-6 signal peptide fused to the N-terminus of NanoLuc, followed by a GSSGAIA linker before the receptor. HaloTag-VEGFR2 and HaloTag-NRP1 were cloned in a pFN21A vector with the IL-6 signal peptide followed by a sequence encoding HaloTag and an EPTTEDLYFQSDNAIA linker at the receptor N-terminus. SnapTagNRP1 was cloned into a pcDNA3.1 vector encoding a murine 5HT3A signal sequence followed by the SnapTag and a STSPVWWNSADIQHSGGRSSGAIA linker. The receptor-encoded sequence from NanoLuc-NRP1 vector was used to generate SnapTag-NRP1 using the Xhol and Xbal restriction sites. N-terminal LgBiT-VEGFR2 and LgBiTNRP1 were cloned in the pFN21A vector with the IL-6 signal peptide, LgBiT sequence, and a flexible GSSGGGGSGGGGSSGGAIA linker. The LgBiT tag sequence from N198A pBiT1.1-N, available from the NanoBiT Multiple Cloning Site Starter System (N2014, Promega Corporation), was cut using Sacll and Sgfl. HiBiT-NRP1 (WT), HiBiT-NRP1 (Y297A), HiBiT-VEGFR2, SmBiT-NRP1 (WT), SmBiT-NRP1 (Y297A), and SmBiT-VEGFR2 were also cloned in a pFN21A vector with the IL-6 signal peptide, 11-amino-acid sequence, and a GSSGGSSGAIA linker. The VEGF-A binding-dead mutant of NRP1 (Y297A) was described previously (Peach, Kilpatrick, et al., 2018). The 11-aminoacid NanoBiT tags (HiBiT: VSGWRLFKKIS; SmBIT VTGYRLFEEIL) were obtained as custom oligonucleotide sequences from SigmaAldrich, annealed into double stranded DNA, and phosphorylated with T4 PNK (New England Biolabs) and inserted using Sacll and Sgfl sites.

\section{3 $\quad$ NFAT luciferase reporter gene assay}

HEK293T-NFAT-ReLuc2P cells stably expressed LgBiT-VEGFR2, HiBiT-VEGFR2, or SmBiT-VEGFR2. Cells were seeded at 25,000 cells per well in white 96-well plates pre-coated with poly-D-lysine in DMEM containing 10\% FBS. Following incubation for $24 \mathrm{~h}$ at $37 \% / 5 \% \mathrm{CO}_{2}$, medium was replaced with serum-free DMEM, and cells were incubated for a further $24 \mathrm{~h}$. On the day of experimentation, medium was replaced with serum-free DMEM containing $0.1 \%$ BSA. Cells were stimulated with increasing concentrations of $V_{E G F_{165}}$ (R\&D Systems) for $5 \mathrm{~h}$ at $37 \% / 5 \% \mathrm{CO}_{2}$. Medium was replaced with $50 \mu \mathrm{l}$ per well serum-free DMEM/0.1\% BSA and $50 \mu \mathrm{l}$ per well ONE-Glo Luciferase reagent. Following a 5-min delay to allow reagent to react with luciferase and background luminescence to subside, luminescence emissions were measured using a TopCount platereader (Perkin Elmer, UK)

\section{4 | Confocal imaging of HaloTag-VEGFR2 and SnapTag-NRP1}

HEK293T-NFAT-ReLuc2P cells were plated in eight-well plates (Nunc Lab-Tek, Thermo Fisher Scientific) pre-coated with poly-D-lysine (0.01 mg. $\mathrm{ml}^{-1}$ in PBS) at 30,000 cells per well in DMEM/10\% FBS. Following incubation for $24 \mathrm{~h}$, cells were transfected with a mixture of HaloTag-VEGFR2 and SnapTag-NRP1. Control wells were also transfected with a single construct and empty vector, such as SnapTag-NRP1 and pcDNA3.1/Neo. Transient transfections used FuGENE® HD at a 3:1 ratio of reagent to CDNA with a total 100-ng cDNA per well, with receptors transfected at equal amounts of 50-ng cDNA per well. Transfection solutions were made up in serum-free DMEM and added as $11 \mu \mathrm{l}$ per well. Cells were incubated for a further $24 \mathrm{~h}$ at $37^{\circ} \mathrm{C} / 5 \% \mathrm{CO}_{2}$. Receptors were then labelled with a solution of serum-free DMEM/0.1\% BSA containing both $0.5-\mu \mathrm{M}$ membraneimpermeant HaloTag-AlexaFluor488 substrate (G1002; Promega Corporation, USA) and 0.5- $\mu \mathrm{M}$ membrane-impermeant SNAP-Surface AlexaFluor647 (S9136S; New England BioLabs). These were incubated for $30 \mathrm{~min}\left(37^{\circ} \mathrm{C} / 5 \% \mathrm{CO}_{2}\right)$. Cells were washed twice with $200 \mu \mathrm{l}$ per well HBSS/0.1\% BSA and then replaced with a final volume of $225 \mu \mathrm{l}$ per well. Cells were incubated with vehicle, 10-nM unlabelled VEGF ${ }_{165} \mathrm{~b}$, or $10-\mathrm{nM}$ unlabelled $\mathrm{VEGF}_{165} \mathrm{a}$ for $60 \mathrm{~min}$ at $37^{\circ} \mathrm{C}$, adding $25 \mu \mathrm{l}$ to a total volume of $250 \mu$ l. Cells were imaged live using a temperature-controlled LSM710 confocal microscope fitted with a 40x water objective (Pan Apochromat objective, NA 1.2). Wavelengths were imaged simultaneously using the 488/561/633 
beamsplitter. HaloTag-VEGFR2 AlexaFluor488 was imaged using an Argon 488-nm laser (493-628 nm of bandpass; $2.5 \%$ power) SnapTag-NRP1 AlexaFluor647 was imaged with a HeNe633-nm laser (638-747 nm; 2.5\% power). All images were taken as 12-bit images with $1024 \times 1024$ pixels per frame with four averages and similar gains per replicate.

\section{5 | Bioluminescence imaging of NanoBiT complexes}

HEK293T-ReLuc2P cells were plated in to poly-D-lysine $\left(0.01 \mathrm{mg} \cdot \mathrm{ml}^{-1}\right.$ in PBS) coated four-chamber $35-\mathrm{mm}$ dishes (10-mm glass coverslip; CellVis Greiner, 627871) at 100,000 cells per quadrant in DMEM/10\% FBS. On day 2, cells were transfected using FuGENE HD at a 3:1 ratio of reagent to cDNA with a total 700-ng cDNA per chamber made up in OptiMEM (ThermoFisher). Cells were transfected with equal amounts of LgBiT-VEGFR2 (350-ng cDNA per chamber) and HiBiT-NRP1 WT (350-ng cDNA per chamber). Alternatively, NanoLuc-VEGFR2 or NanoLuc-NRP1 was transfected at 350-ng cDNA per chamber with an equal amount of pcDNA3.1/Zeo (350-ng cDNA per chamber). On day 3, medium was replaced with $\mathrm{HBSS} / 0.1 \%$ BSA containing furimazine $(26 \mu \mathrm{M})$. Following incubation for $10 \mathrm{~min}$ to allow for substrate oxidation, cells were imaged live at $37^{\circ} \mathrm{C}$ using the inverted Olympus LV200 Bioluminescence Imaging System, fitted with a $60 \times$ oil immersion objective (super Apochromat UPLSAPO $60 \times 0$ objective; NA 1.35 ) with a $0.5 \times$ tube lens to focus the image; therefore, images had a final magnification of $30 \times$. Luminescence was collected using a Hamamatsu Image EMx2 Electron Multiplying Charge Coupled Device (EMCCD) camera. Transmitted light images were collected using the camera in conventional CCD mode with a 250-ms exposure time. Luminescence emissions from the full-length NanoLuc or the NanoBiT complex were measured for 10-s exposure with a gain of 15-30. Images were taken as 8-bit images with $512 \times 512$ pixels per frame.

\section{6 | BRET between NanoLuc-VEGFR2 and fluorescent NRP1}

HEK293T-NFAT-ReLuc2P cells were plated in white 96-well plates pre-coated with poly-D-lysine $\left(0.01 \mathrm{mg} \cdot \mathrm{ml}^{-1}\right.$ in PBS) at 25,000 cells per well in DMEM containing 10\% FBS. Following $24 \mathrm{~h}$, cells were transiently transfected with a total 125-ng cDNA per well using FuGENE HD at a 3:1 ratio of reagent to cDNA. Cells were transfected with a constant amount of NanoLuc-VEGFR2 (25-ng cDNA per well). Cells were simultaneously transfected with increasing concentrations of HaloTag-NRP1 or SnapTag-NRP1 (2.5- to 100-ng cDNA per well). Additional wells only contained NanoLuc-VEGFR2. These transfection solutions were made up to equivalent to $125 \mathrm{ng}$ per well using empty pcDNA3.1/Zeo vector in serum-free DMEM. Cells were incubated for another $24 \mathrm{~h}$ at $37^{\circ} \mathrm{C} / 5 \% \mathrm{CO}_{2}$. On the day of the experiment, cells were treated with $0.2-\mu \mathrm{M}$ membrane-impermeant HaloTag-
AlexaFluor488 substrate or $0.2-\mu \mathrm{M}$ SNAP-Surface AlexaFluor488 substrate in serum-free DMEM/0.1\% BSA. Cells were incubated for $30 \mathrm{~min}$ at $37^{\circ} \mathrm{C} / 5 \% \mathrm{CO}_{2}$. They were then washed twice with $100 \mu \mathrm{l}$ per well HBSS $/ 0.1 \%$ BSA and replaced with a final volume of $50 \mu \mathrm{l}$ per well HBSS/0.1\% BSA. At this stage, fluorescence emissions were quantified using the PHERAstar FS platereader using filters for excitation at $485 \mathrm{~nm}$ and emission at $520 \mathrm{~nm}$. Cells were then incubated with the NanoLuc substrate furimazine $(10 \mu \mathrm{M})$ for $5 \mathrm{~min}$. Emissions were recorded using the PHERAstar FS platereader using filters simultaneously measuring NanoLuc emissions at $475 \mathrm{~nm}$ (30-nm bandpass) and AlexaFluor488 emissions at $535 \mathrm{~nm}$ (30-nm bandpass). BRET ratios were calculated as fluorescence over luminescence emissions from the second of three cycles.

\section{7 | Luminescence from NanoBiT complementation}

To characterise luminescence emissions from a NanoBiT complex, HEK293T-NFAT-ReLuc2P cells were plated as 25,000 cells per well in white 96-well plates pre-coated with poly-D-lysine $\left(0.01 \mathrm{mg} \cdot \mathrm{ml}^{-1}\right.$ in PBS) in DMEM containing 10\% FBS. Following $24 \mathrm{~h}$, cells were transiently transfected using FuGENE HD at a 3:1 ratio of reagent to cDNA with a total 100-ng cDNA per well. Cells were transfected with a combination of LgBiT-tagged (50-ng cDNA per well) and HiBiT-/ SmBiT-tagged receptors (50-ng cDNA per well). Alternatively, cells were transfected with single constructs (50-ng cDNA per well) with empty pcDNA3.1/Zeo vector (50-ng cDNA per well). Transfection mixtures were made up in serum-free DMEM and added as $5 \mu \mathrm{l}$ per well without replacing DMEM/10\% FBS on cells. Cells were incubated at $37^{\circ} \mathrm{C} / 5 \% \mathrm{CO}_{2}$ for a further $24 \mathrm{~h}$. Medium was replaced with $\mathrm{HBSS} / 0.1 \%$ BSA containing $10-\mu \mathrm{M}$ furimazine, in the absence or presence of purified LgBiT protein (N401B, Promega Corporation) or HiBiT protein (N301A, Promega Corporation). Cells were incubated at $37^{\circ} \mathrm{C}$ for $10 \mathrm{~min}$ to allow NanoBiT complementation and the oxidation of furimazine. To prevent the loss of signal through the bottom of the plate, an adhesive plate BackSeal was added at this point. Luminescence emissions were measured on the PHERAstar platereader using the filter settings measuring emissions between 475 and $505 \mathrm{~nm}$.

Additional experiments aimed to disrupt the recomplemented NanoBiT complex using increasing concentrations of competing receptor. HEK293T-NFAT-ReLuc2P cells were plated as 25,000 cells per well in white 96-well plates pre-coated with poly-D-lysine (0.01 $\mathrm{mg} \cdot \mathrm{ml}^{-1}$ in PBS) in DMEM containing 10\% FBS. Following $24 \mathrm{~h}$, cells were transiently transfected using FuGENE HD at a 3:1 ratio of reagent to cDNA. Cells were transfected with a constant amount of LgBiT-VEGFR2 (50-ng cDNA per well) and either HiBiT-NRP1 or SmBiT-NRP1 (50-ng cDNA per well). Cells were also transfected with increasing concentrations of HaloTag-NRP1 (25- to 200-ng cDNA per well). This was made up to 300-ng cDNA per well with empty pcDNA3.1/Zeo vector. Additional wells only contained the LgBiT-VEGFR2 and HiBiT/SmBiT-NRP1 complex. Cells were incubated with transfection solution for $24 \mathrm{~h}$ at $37^{\circ} \mathrm{C} / 5 \% \mathrm{CO}_{2}$. On the day 
of the experiment, cells were treated with $0.2-\mu \mathrm{M}$ membraneimpermeant HaloTag-AlexaFluor488 substrate in serum-free DMEM/0.1\% BSA (30 min, $37^{\circ} \mathrm{C} / 5 \% \mathrm{CO}_{2}$ ). They were then washed twice with $100 \mu \mathrm{l}$ per well HBSS/0.1\% BSA and replaced with a final volume of $50 \mu \mathrm{l}$ per well HBSS/0.1\% BSA. Fluorescence emissions were quantified using the PHERAstar FS platereader using filters for excitation at $485 \mathrm{~nm}$ and emission at $520 \mathrm{~nm}$. Cells were incubated with $10-\mu \mathrm{M}$ furimazine for $10 \mathrm{~min}$; then luminescence and fluorescence emissions were recorded using PHERAstar FS platereader. Emissions were simultaneously measured for NanoLuc at $475 \mathrm{~nm}$ (30-nm bandpass) and AlexaFluor488 at $535 \mathrm{~nm}$ (30-nm bandpass).

\section{8 | Fluorescent VEGF-A binding at a VEGFR2/ NRP1 NanoBiT complex}

HEK293T-NFAT-ReLuc2P cells were plated in six-well plates at 400,000 cells per well in DMEM containing $10 \%$ FBS. On day 2 , cells were transfected using FuGENE HD at a 3:1 ratio of reagent to cDNA with a total 1,500-ng cDNA per well made up in serum-free DMEM. Cells were transfected with equal amounts of LgBiT-VEGFR2 (750-ng cDNA per well) and HiBiT-NRP1 WT or Y297A (750-ng cDNA per well) or equal amounts of LgBiT-VEGFR2 (750-ng cDNA per well) with SmBiT-NRP1 WT (750-ng cDNA per well). For experiments monitoring kinetics at $\mathrm{HiBiT}$ complexes, matched controls were performed alongside in which cells were transfected with single receptors conjugated to full-length NanoLuc. NanoLuc-VEGFR2 or NanoLuc-NRP1 was transfected at 750-ng cDNA per well, made up to 1,500-ng cDNA per well with empty pcDNA3.1/Zeo vector (750-ng cDNA per well). On day 3, cells were transferred from sixwell plates. Cells were washed with $1 \mathrm{ml}$ per well of PBS, detached with $500 \mu \mathrm{l}$ per well of trypsin, and resuspended in 2-ml DMEM containing $10 \%$ FBS. Cells were seeded in white $96-$ well plates precoated with poly-D-lysine $\left(0.01 \mathrm{mg} \cdot \mathrm{ml}^{-1}\right.$ in PBS) at 30,000 cells per well. On the day of experimentation (day 4), medium was replaced with $\mathrm{HBSS} / 0.1 \%$ BSA.

For saturation experiments, increasing concentrations of VEGF $_{165}$ a-TMR or VEGF 165 b-TMR $(0.5-20 \mathrm{nM})$ were added in the presence or absence of a high concentration of corresponding unlabelled ligand $\left(100 \mathrm{nM}, \sim 100\right.$-fold greater than the estimated $K_{d}$ value). Following incubation for $60 \mathrm{~min}$ in the dark at $37^{\circ} \mathrm{C}$, the NanoLuc substrate furimazine $(10 \mu \mathrm{M})$ was added to each well and equilibrated for $5 \mathrm{~min}$ to enable NanoLuc-mediated furimazine oxidation and resulting luminescence emissions. Emissions were recorded using the PHERAstar FS platereader (BMG Labtech) using a filter simultaneously measuring NanoLuc emissions at 450 nm (30-nm bandpass) and TMR emissions using a longpass filter at $550 \mathrm{~nm}$. BRET ratios were calculated as fluorescence over luminescence emissions from the second of three cycles.

For kinetic experiments, cells were pretreated with furimazine $(10 \mu \mathrm{M})$ for $5 \mathrm{~min}$ to enable NanoLuc-mediated furimazine oxidation and resulting luminescence emissions. BRET ratios were then measured per well using the PHERAstar FS platereader using the filters above. Following four initial measurements, intact cells were stimulated with $0.5-20 \mathrm{nM}$ of VEGF $_{165} \mathrm{a}-\mathrm{TMR}$ or VEGF $165 \mathrm{~b}-\mathrm{TMR}$. Emissions were recorded every $30 \mathrm{~s}$ for 20 or $90 \mathrm{~min}$, using the temperature control function of the PHERAstar FS platereader to maintain conditions at $37^{\circ} \mathrm{C}$.

\subsection{Data analysis}

Data were analysed using GraphPad Prism 7.02 (GraphPad Software, La Jolla, CA, USA; RRID:SCR_002798). Data are presented as mean \pm SEM. All experiments were performed in three to six independent experiments with duplicate or triplicate wells (see figure legends for details). Drug additions were randomly allocated to wells within each 96-well plate. Statistical significance was defined as $P<.05$. Confocal images were collected using Zen 2010 software (Zeiss, Germany). Confocal images were processed and analysed using ImageJ Fiji 1.52 software (National Institutes of Health, USA; RRID: SCR_003070). The data and statistical analysis comply with the recommendations of the British Journal of Pharmacology on experimental design and analysis in pharmacology.

For co-localisation analysis, confocal images were corrected to the background fluorescence intensity from each experimental replicate determined using untransfected cells in each field of view (HaloTag-VEGFR2, 488 nm; SnapTag-NRP1, 647 nm). The mean background intensity was calculated for each experimental replicate $(n=6)$ and subtracted from each image for manual thresholding. To quantify co-localisation, regions of interest (ROIs) were drawn around each cell that co-expressed HaloTag-VEGFR2 and SnapTag-NRP1. Following subtraction of the region outside the ROI, co-localisation was determined using pixel-based measures between HaloTag-VEGFR2 and SnapTag-NRP1 using the ImageJ plugin Coloc 2. Mander's overlap coefficients measure co-occurrence as the proportion of SnapTag-NRP1 pixels (red) overlapping with HaloTag-VEGFR2 (green). Pearson's correlation coefficients measure whether there is a correlation between these channels. Co-localisation parameters were calculated on a per cell basis, with a total number of 119 cells (vehicle), 78 cells $\left(\mathrm{VEGF}_{165}\right.$ a stimulation), and 93 cells $\left(\mathrm{VEGF}_{165} \mathrm{~b}\right.$ stimulation), pooled from six independent experiments. Blinding was not performed for these imaging experiments because of the complexity of the experimental design. However, Coloc 2 analysis was performed automatically on all cells in every field of view that co-expressed HaloTag-VEGFR2 and SnapTag-NRP1.

Saturation binding curves were fitted simultaneously for total (VEGF $_{165}$ a-TMR or VEGF VE5 $_{16}$-TMR alone) and non-specific binding (obtained in the presence of $100 \mathrm{nM}$ of unlabelled VEGF-A) using the equation:

$$
\text { Total Binding }=B_{\max } \cdot \frac{[L]}{[L]+K_{d}}+M \cdot[L]+C
$$

describing the nanomolar fluorescent ligand concentration, [L]; maximal specific binding, $B_{\max }$; the equilibrium dissociation constant of the 
labelled ligand, $K_{d}$, in the same units as [L]; the slope of the nonspecific binding component, $M$; and the $y$-axis intercept, $c$.

Association kinetic studies were performed with four concentrations of ligand simultaneously for global fitting in order to determine the $k_{\text {on }}$ and $k_{\text {off }}$. Kinetic studies of fluorescent ligand binding measured over time were fitted to a mono-exponential association function:

$$
\text { Binding }=y_{\max } \cdot\left(1-e^{-k_{\text {obs }} \cdot t}\right)
$$

describing time, $t$, plotted on the $x$ axis; maximum response at infinite time, $y_{\max }$; and the rate constant observed for association, $k_{\text {obs. }}$. Additionally, $k_{\text {on }}$ and $k_{\text {off }}$ values were determined by simultaneously fitting association curves at different fluorescent ligand concentrations ([L]). This utilised the following relationship with $k_{\text {obs: }}$ :

$$
k_{\mathrm{obs}}=k_{\mathrm{on}} \cdot[L]+k_{\mathrm{off}}
$$

further describing association rate, $k_{\mathrm{on}}$, in units of $\min ^{-1} \cdot \mathrm{M}^{-1}$; and dissociation rate, $k_{\text {off }}$, in $\min ^{-1}$. GraphPad Prism was used to fit each association curve to the above equations with the parameters for $k_{\text {on }}$ and $k_{\text {off }}$ shared between the fits for the four different concentrations of fluorescent ligand used in each experiment. This allowed values for $k_{\text {on }}$ and $k_{\text {off }}$ values to be determined for each experiment. These kinetic data were also used to estimate the binding affinities, due to the relationship between dissociation and association rates within an equilibrium:

$$
K_{d}=\frac{k_{\text {off }}}{k_{\text {on }}}
$$

\subsection{0 | Materials}

Fluorescent $\mathrm{VEGF}_{165} \mathrm{a}$ and $\mathrm{VEGF}_{165} \mathrm{~b}$ were labelled at a single $\mathrm{N}$-terminal cysteine residue with TMR using the HaloTag mammalian protein detection and purification system (G6795; Promega Corporation, USA) as described previously (Kilpatrick et al., 2017; Peach, Kilpatrick et al., 2018). Fluorescent ligands were characterised in terms of labelling efficiency, dimerisation, and function as described in Kilpatrick et al. (2017) and Peach, Kilpatrick et al. (2018). Ligands were stored at $-20^{\circ} \mathrm{C}$ in $2.5 \mathrm{mg} \cdot \mathrm{ml}^{-1}$ protease-free BSA (Millipore, USA). Unlabelled recombinant human VEGF isoforms were purchased from R\&D Systems (Abingdon, UK). Furimazine and purified NanoBiT fragments were purchased from Promega Corporation (Madison, USA).

\subsection{Nomenclature of targets and ligands}

Key protein targets and ligands in this article are hyperlinked to corresponding entries in http://www.guidetopharmacology.org and are permanently archived in the Concise Guide to PHARMACOLOGY 2019/20 (Alexander et al., 2019).

\section{3 | RESULTS}

\section{1 | Co-localisation between VEGFR2 and NRP1 co-expressed in living HEK293T cells}

To investigate where VEGFR2 and NRP1 were localised when both receptors were expressed together in HEK293T cells at $37^{\circ} \mathrm{C}$, we labelled each cell surface receptor with a distinct fluorophore. Receptors were simultaneously labelled using different substrates containing a HaloTag chloroalkane or SnapTag benzylguanine moiety, exploiting the fact that the membrane-impermeant fluorophoreconjugated substrate only labels receptors at the plasma membrane. HaloTag-VEGFR2 and SnapTag-NRP1 were labelled with membraneimpermeant HaloTag-AlexaFluor488 and SnapTag-AlexaFluor647 (Figure 1a). Constitutive internalisation of HaloTag-VEGFR2 was observed (Figure 1a, green regions) whereas SnapTag-NRP1 was largely expressed at the plasma membrane (Figure 1a, magenta regions). Sites of spatial overlay between VEGFR2 and NRP1 were both intracellular and at regions around the plasma membrane (Figure 1a, white). The same cell population was stimulated with a saturating concentration of unlabelled $\mathrm{VEGF}_{165} \mathrm{~b}$ (upper panels) or VEGF $_{165}$ a (lower panels) for $60 \mathrm{~min}$ (Figure 1a, right panels). Representative images show a large proportion of NRP1 remained at the plasma membrane independent of VEGF-A stimulation. To account for heterogeneity between cells, ROls were drawn around any cell successfully co-expressing both RTK and co-receptor to quantify co-localisation between HaloTag-VEGFR2 and SnapTagNRP1. Upon stimulation with VEGFR2-selective $V_{E G F} 165 \mathrm{~b}$, there was a reduction in the proportion of NRP1 in VEGFR2-positive regions relative to vehicle (Figure $1 \mathrm{~b}$ ). In contrast, there was a higher correlation between VEGFR2/NRP1 co-localisation upon VEGF 165 a stimulation compared to vehicle (Figure 1c). Both parameters indicated that VEGFR2 and NRP1 were co-localised in the absence of ligand.

BRET can also be applied to monitor proximity between receptors tagged with a bioluminescent donor (NanoLuc) and fluorescent acceptor (AlexaFluor488). Receptor-receptor BRET was used to monitor whether VEGFR2 and NRP1 were in proximity $(<10 \mathrm{~nm})$ when co-expressed in HEK293T cells. This unbiased technique monitors proximity from a whole cell population in 96-well plates. Cells were simultaneously transfected with a constant amount of bioluminescent donor, NanoLuc-VEGFR2, and increasing amounts of cell surface fluorophore-labelled NRP1. In the absence of ligand, there was clear saturation of the BRET signal with increasing amounts of fluorescent NRP1 acceptor (Figure 2a). This was observed for both SnapTagNRP1 and HaloTag-NRP1, therefore independent of the fluorophore labelling approach. Confirming that increasing amounts of HaloTagNRP1 and SnapTag-NRP1 were successfully transfected, there was also a saturable BRET signal when plotted against raw fluorescence emissions (Figure $2 b$ ). These complementary data were obtained using the same technique to confirm that increased fluorescent protein led to saturation of the BRET signal. Both the confocal imaging studies (Figure 1) and the saturation BRET studies (Figure 2) provided 
(a)
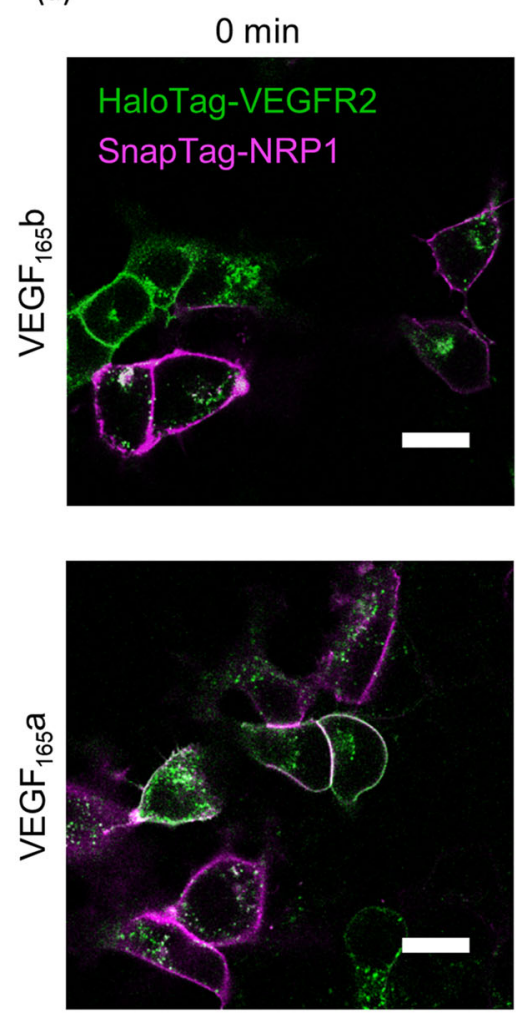

(b)
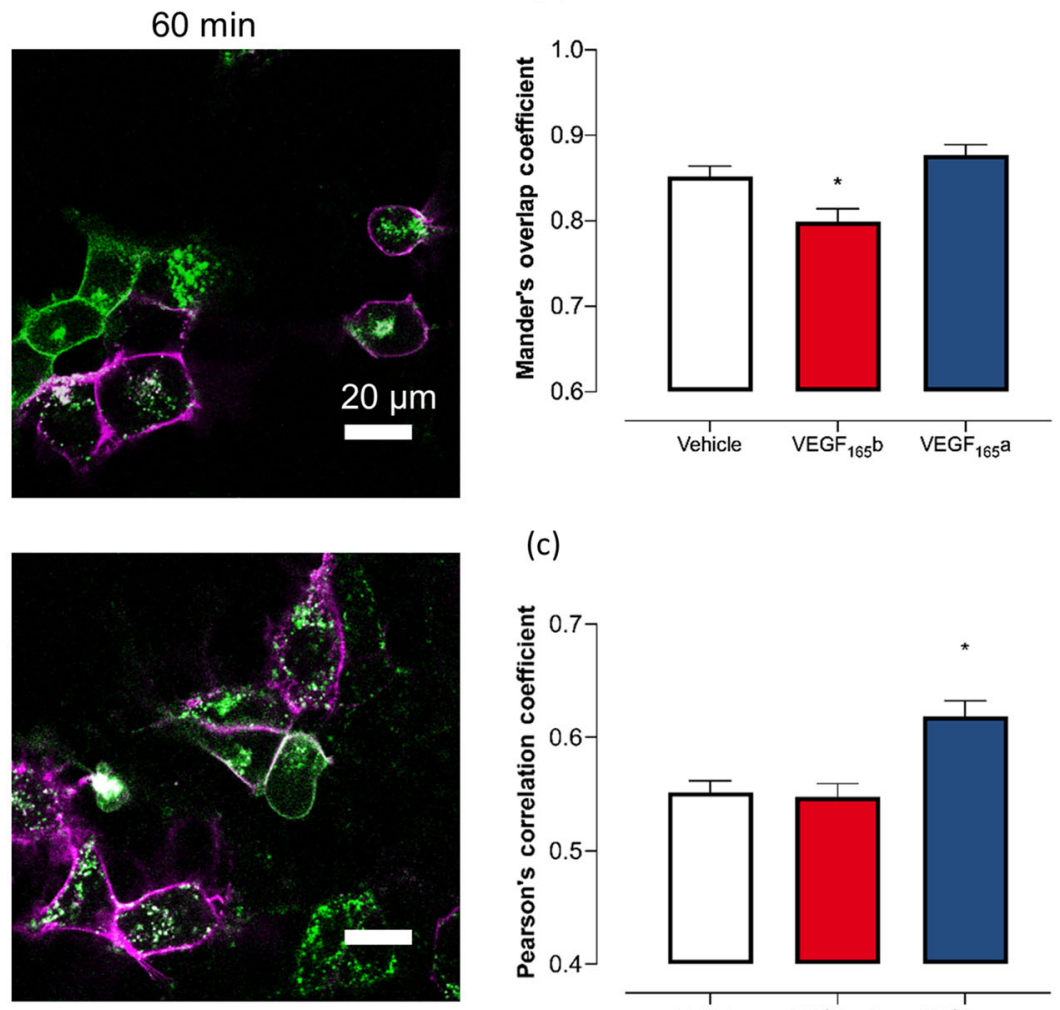

(c)

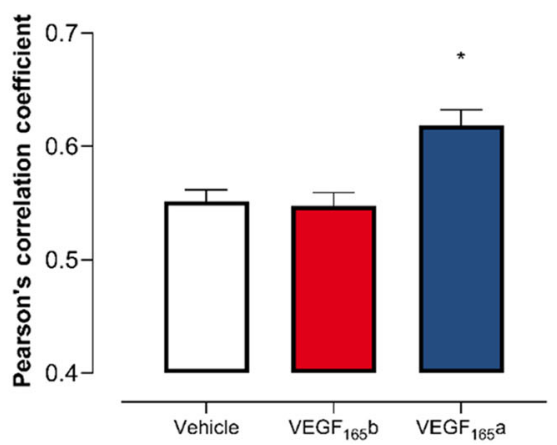

FIGURE 1 Localisation of VEGFR2 and NRP1 co-expressed in living HEK293T cells using confocal microscopy. (a) HEK293T cells expressing HaloTag-VEGFR2 and SnapTag-NRP1 were simultaneously labelled with 0.5- $\mu$ M membrane-impermeant HaloTag-AlexaFluor488 and 0.5- $\mu \mathrm{M}$ SnapTag-AlexaFluor647 for $30 \mathrm{~min}\left(37^{\circ} \mathrm{C}\right)$. Cells were washed twice in HEPES buffered saline solution (HBSS) containing $0.1 \%$ BSA and incubated at $37^{\circ} \mathrm{C}$. Cells were imaged on the LSM710 confocal microscope (40x objective). The same cell population were imaged before (left panel) and after (right panel) treatment with 10-nM unlabelled VEGF $_{165} \mathrm{~b}$ or $\mathrm{VEGF}_{165^{2}}$ for 60 min ( $37^{\circ} \mathrm{C}$ ). Images show HaloTag-VEGFR2 (green) and SnapTag-NRP1 (magenta), showing regions of spatial overlay in white. Images are representative of six independent experiments. (b,c) ImageJ/Fiji software was used to analyse images with channels corresponding to HaloTag-VEGFR2 or SnapTag-NRP1. Co-localisation was quantified based on regions of interest drawn around cells co-expressing both receptors. Mander's overlap coefficients represent the proportion of SnapTag-NRP1 in HaloTag-VEGFR2+ regions (b), whereas Pearson's correlation coefficients compare the relationship between the intensity of VEGFR2 and NRP1 pixels (c). All coefficient values were pooled from six independent experiments, with a total of 119 cells (vehicle), 93 cells $\left(V_{16 F_{165}} \mathrm{~b}\right)$, or 78 cells $\left(\mathrm{VEGF}_{165} \mathrm{a}\right)$. Coefficients were compared between conditions using a Kruskal-Wallis test and post hoc Dunn's multiple comparisons test between vehicle, $V_{E G F} 165$, or $V_{E G F} F_{165}$ a stimulation. ${ }^{*} P<.05$, significantly different from vehicle and $\mathrm{VEGF}_{165}$ a or $\mathrm{VEGF}_{165} \mathrm{~b}$

evidence for the constitutive formation of heteromeric complexes between VEGFR2 and NRP1 in living cells.

\subsection{Complementation of NanoBiT fragments using N-terminal tagged VEGFR2 and NRP1}

We then applied a split NanoBiT approach to isolate luminescence emissions from a defined VEGFR2/NRP1 heteromeric complex. Enzymic luciferase activity requires complementation between the large fragment (LgBiT) and the short 11-amino-acid tag (HiBiT or SmBiT). To determine the optimal configuration for luminescence emissions, each NanoBiT fragment was appended to the $\mathrm{N}$-terminus of both full-length VEGFR2 and NRP1. Luminescence emissions were higher for the combination with LgBiT-tagged VEGFR2 and the short fragment attached to NRP1 (Figure 3a). Emissions from the HiBiT complex were approximately 10-fold higher than the SmBiT complex. NanoBiT-tagged receptors expressed independently emitted minimal luminescence in the presence of furimazine relative to the complemented NanoBiT complex (Figure 3b). Addition of purified NanoBiT fragments to exogenously complement the NanoBiT tag confirmed that individual constructs were appropriately expressed despite low luminescence emissions in isolation (Figure 3c). The luminescence signals from both $\mathrm{HiBiT}$ and $\mathrm{SmBiT}$ complexes were also prevented by competition with increasing amounts of unlabelled HaloTag NRP1 (Figure 3d). Thus, despite the intrinsic affinity between HiBiT and LgBiT (Dixon et al., 2016), luminescence emissions were reduced by increasing amounts of NRP1.

A bioluminescence widefield imaging system was used to visualise where the NanoBiT luminescence signal was localised. To determine the cellular location of the luciferase signal, cells were incubated with membrane-permeable furimazine in the absence of ligand 
(a)

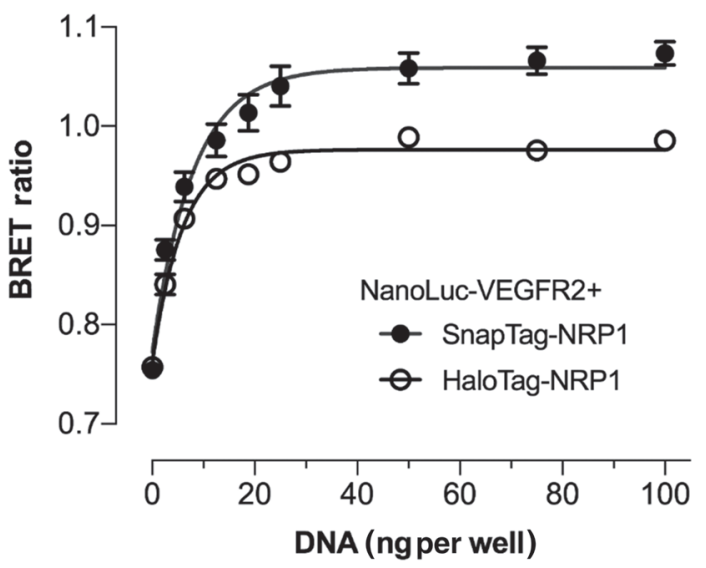

(b)

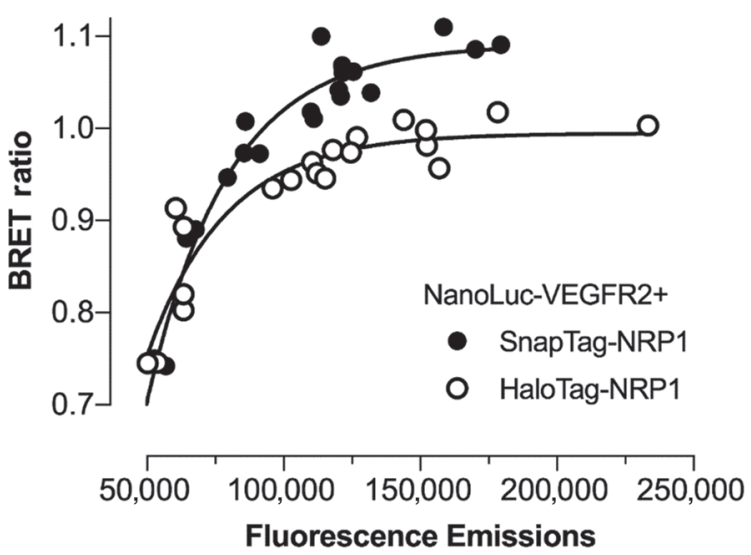

FI G URE 2 Oligomer formation between VEGFR2 and NRP1. (a,b) HEK293T cells were transiently transfected with a fixed concentration of NanoLuc-VEGFR2 (25-ng cDNA per well) and increasing concentrations of fluorescent acceptor (HaloTag-NRP1 or SnapTag-NRP1, 0- to 100-ng cDNA per well). All wells were transfected with 125-ng cDNA per well total with empty pcDNA3.1/Zeo vector. NRP1 was labelled with 0.2- $\mu$ M HaloTag-AlexaFluor488 substrate or $0.2-\mu \mathrm{M}$ SNAP-Surface AlexaFluor488 substrate for $30 \mathrm{~min}\left(37^{\circ} \mathrm{C}\right)$. Cells were washed twice with HBSS/0.1\% $\mathrm{BSA}$ and then incubated in $10-\mu \mathrm{M}$ furimazine for $5 \mathrm{~min}\left(37^{\circ} \mathrm{C}\right)$. Emissions from the luminescent donor and fluorescent acceptor receptor were simultaneously monitored by the PHERAstar FS platereader. Data are expressed as (a) mean \pm SEM from five independent experiments with duplicate wells or (b) individual data points from a representative experiment plotting BRET ratio values against fluorescence emissions $(485-520 \mathrm{~nm})$

(Figure 4). NanoLuc-VEGFR2 was largely intracellular, whereas NanoLuc-NRP1 was at the cell surface. The NanoBiT complex between HiBiT-NRP1 and LgBiT-VEGFR2 was localised to both intracellular sites and the plasma membrane. This spatial distribution was comparable to the regions of white overlay between HaloTagVEGFR2 and SnapTag-NRP1 observed in Figure 1a.

\section{3 | Influence of NanoBiT tags on VEGFR2-mediated signalling}

We confirmed that the NanoBiT fragments did not interfere with VEGFR2 signalling using an NFAT reporter gene assay (Kilpatrick et al., 2017). Concentration-response curves for $V_{E G F}$ 165 a were compared between cells stably expressing VEGFR2 tagged at the $\mathrm{N}$-terminus with $\mathrm{LgBiT}$, HiBiT, or SmBiT (Figure 5). Each receptor exhibited a concentration-dependent increase in NFAT gene transcription in response to increasing concentrations of VEGF $_{165}$ a. Each cell line had a similar potency derived for $\mathrm{VEGF}_{165}{ }^{a}$ (LgBiT-VEGFR2 $\quad \mathrm{pEC}_{50}=9.95 \pm 0.11 ; \quad$ HiBiT-VEGFR2 $\mathrm{pEC}_{50}=10.06 \pm 0.12 ;$ SmBiT-VEGFR2 $\mathrm{pEC}_{50}=10.23 \pm 0.23 ; n=5$ for each). These were comparable to potency values derived for VEGF $_{165}$ a at wild-type VEGFR2 (Kilpatrick et al., 2017).

\subsection{Nanomolar affinity of fluorescent VEGF-A at a defined VEGFR2/NRP1 complex}

Fluorescent VEGF-A ligand binding was monitored at full-length VEGFR2 and NRP1 tagged at their $\mathrm{N}$-terminus with LgBiT and
$\mathrm{HiBiT}$, respectively. As the uncomplemented receptors cannot oxidise furimazine, luminescence was confined to proteins where complementation from a defined heteromeric VEGFR2/NRP1 NanoBiT complex had occurred (Figure 6a). BRET therefore only derived from the receptor/co-receptor complex and the fluorescent VEGF-A acceptor. We have previously demonstrated that VEGF $_{165} \mathrm{~b}-\mathrm{TMR}$ selectively binds to NanoLuc-VEGFR2 (and not NRP1), whereas VEGF $_{165}$ a-TMR can bind to both NanoLuc-VEGFR2 and NanoLucNRP1 with nanomolar affinity (Peach, Kilpatrick, et al., 2018). At the complemented HiBiT complex, there was saturable binding in the presence of increasing concentrations of VEGF $165 \mathrm{~b}-\mathrm{TMR}$ (Figure $6 \mathrm{~b}$ ) or VEGF 165 a-TMR (Figure 6c). This was displaced by a high concentration of unlabelled ligand, demonstrating low non-specific binding. Both fluorescent ligands had equilibrium dissociation constants $\left(K_{d}\right)$ in the nanomolar range at the VEGFR2/NRP1 complex $\left(\mathrm{VEGF}_{165} \mathrm{~b}\right.$ TMR $\quad K_{d}=16.26 \pm 3.81 \mathrm{nM}, \quad \mathrm{pK} K_{d}=7.82 \pm 0.11 ; \quad V^{2} G_{165} \mathrm{a}-\mathrm{TMR}$ $K_{d}=2.53 \pm 0.49, p K_{d}=8.61 \pm 0.09 ; n=3$ for both). Estimated ligand binding affinities were similar to those derived at isolated receptors tagged with full-length NanoLuc (Peach, Kilpatrick, et al., 2018).

\section{5 | Real-time kinetics of fluorescent VEGF-A isoforms at a heteromeric VEGFR2/NRP1 NanoBiT complex}

Taking advantage of the NanoBiT approach to monitor real-time ligand binding at $37^{\circ} \mathrm{C}$ to a complex, we compared the kinetics of ligand binding of VEGF 165 b-TMR with that of VEGF 165 a-TMR at the VEGFR2/NRP1 NanoBiT complex in living cells. The kinetic binding profile of VEGF ${ }_{165}$ b-TMR (which should only bind to VEGFR2; Peach, 
(a)

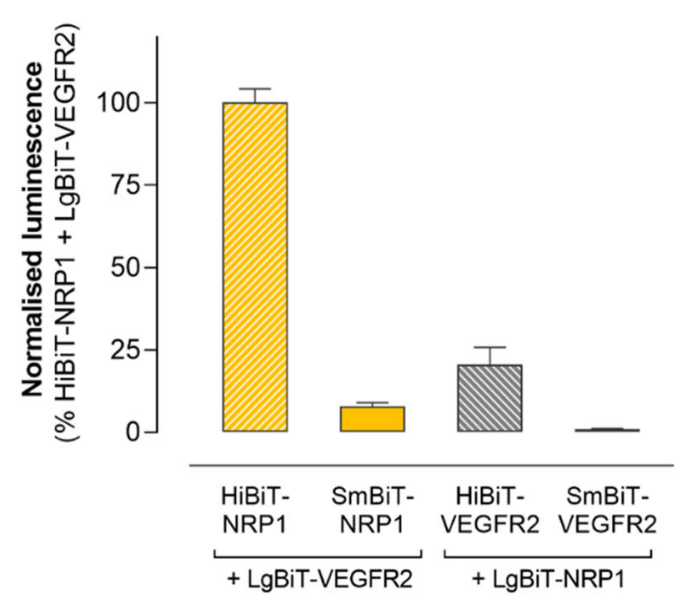

(c)

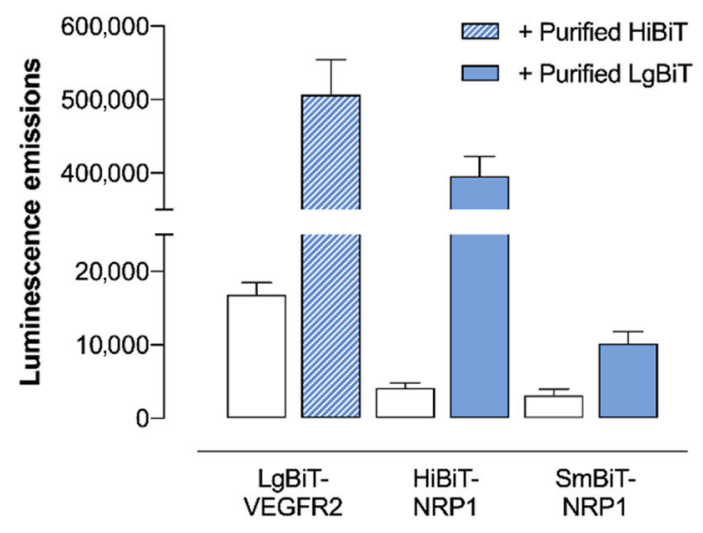

(b)

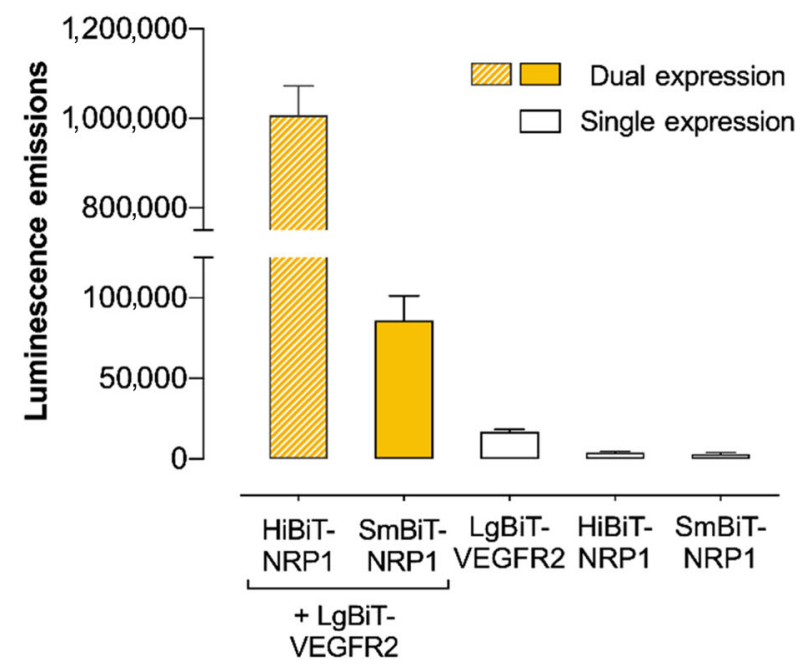

(d)

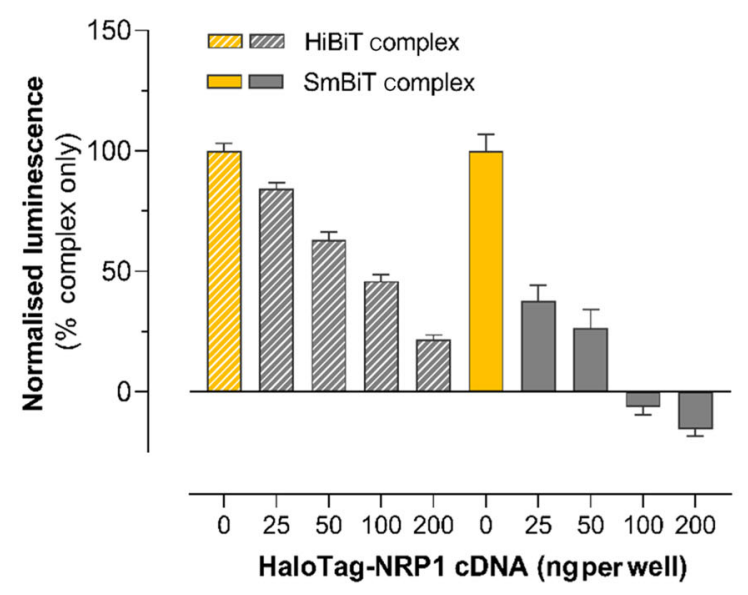

FIGURE 3 Complementation of a VEGFR2/NRP1 NanoBiT complex. (a) To determine the optimal orientation of labelling with NanoLuc Binary Technology (NanoBiT) fragments, each receptor was tagged with the 18-kDa fragment (LgBiT) and a smaller 11-amino-acid fragment. $\mathrm{HiBiT}$ has a higher intrinsic affinity to complement with LgBiT compared to SmBiT (Dixon et al., 2016). HEK293T cells were transiently transfected in 96-well plates with equal amounts of LgBiT-tagged receptor (50-ng cDNA per well) and HiBiT- or SmBiT-tagged receptor (50-ng cDNA per well). Cells were incubated with $10-\mu \mathrm{M}$ furimazine in $\mathrm{HBSS} / 0.1 \%$ BSA for $10 \mathrm{~min}\left(37^{\circ} \mathrm{C}\right)$. Data were normalised to untransfected cells (0\%) and HiBiT-NRP1/LgBiT-VEGFR2 (100\%) per experiment. Data are expressed as mean \pm SEM from five independent experiments (LgBiTVEGFR2) or three independent experiments (LgBiT-NRP1), each with triplicate wells. (b) To compare emissions from individual NanoBiT-tagged receptors relative to a complemented NanoBiT complex, HEK293T cells were transiently transfected in 96-well plates with LgBiT-VEGFR2, HiBiT-NRP1, or SmBiT-NRP1 (50-ng cDNA per well). Dual expression cells expressed a complemented NanoBiT complex (filled bars) whereas single constructs (empty bars) were transfected with 50-ng cDNA per well of empty pcDNA3.1/Zeo vector for 100-ng total cDNA per well. Raw luminescence emissions were plotted as mean \pm SEM from five independent experiments. (c) Cells expressed a single NanoBiT-tagged construct, with or without purified HiBiT or LgBiT (20-nM). Raw emissions were plotted as mean \pm SEM from five independent experiments. (d) Prevention of NanoBiT complex formation by co-expression of increasing amounts of competing VEGFR2 or NRP1. HEK293T cells were transfected with equal amounts of LgBiT-VEGFR2 (50-ng cDNA per well) and either HiBiT-NRP1 or SmBiT-NRP1 at 50-ng cDNA per well. Cells were also transfected with increasing amounts of HaloTag-NRP1 (0- to 200-ng cDNA per well), as well as with pcDNA3.1/Zeo empty vector (for 300-ng total cDNA per well). Data were normalised to untransfected cells $(0 \%)$ and the complemented NanoBiT complex in the absence of competing receptor (100\%) per experiment. Data are expressed as mean \pm SEM from three independent experiments, each with triplicate wells. In each experiment $(a-d)$, cells were incubated with furimazine $(10 \mu \mathrm{M})$ in HBSS $/ 0.1 \%$ BSA for 10 min $\left(37^{\circ} \mathrm{C}\right)$. Luminescence emissions $(475-505 \mathrm{~nm})$ were measured by the PHERAstar FS platereader

Kilpatrick, et al., 2018) continued to increase over the full 90-min time course in intact cells, producing a classic ligand binding association maintained for each concentration of $\mathrm{VEGF}_{165} \mathrm{~b}$-TMR (Figure 7a).
Fitted to a global association curve (Table 1), VEGF VE5 $_{165}$-TMR had a slightly slower association rate constant $\left(k_{\text {on }}\right)$ for the VEGFR2/ NRP1 complex $\left(2.29 \times 10^{6} \pm 0.30 \times 10^{6} \mathrm{~min}^{-1} \cdot \mathrm{M}^{-1}\right)$ compared to 
VEGFR2/NRP1 complex

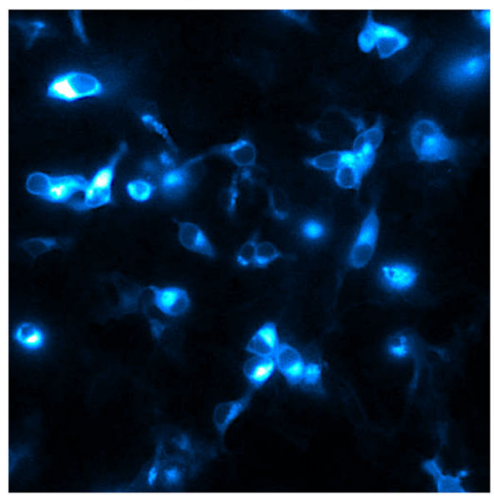

NanoLuc-VEGFR2

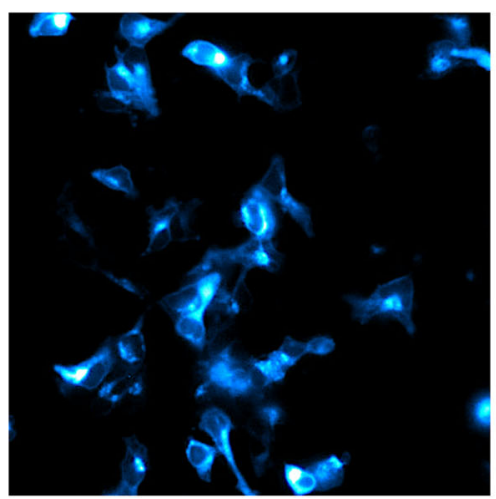

NanoLuc-NRP1

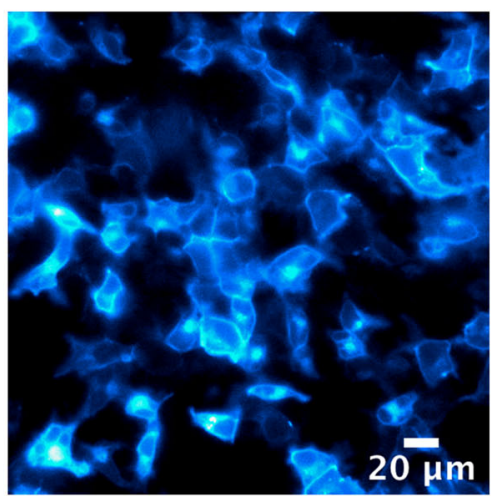

FIG URE 4 Bioluminescence imaging of cells expressing NanoBiT-complemented VEGFR2-NRP1 complexes, NanoLuc-VEGFR2, or NanoLucNRP1. HEK293T cells were seeded in four-chamber $35-\mathrm{mm}^{2}$ glass-bottomed dishes. The following day, cells were transfected with LgBiTVEGFR2 (350-ng cDNA per chamber) and HiBiT-NRP1 (350-ng cDNA per chamber). Alternatively, cells were transfected with NanoLuc-VEGFR2 or NanoLuc-NRP1 (350-ng cDNA per chamber) with pcDNA3.1/Zeo (250-ng cDNA per chamber). Following 24 h, cells were incubated in $\mathrm{HBSS} / 0.1 \% \mathrm{BSA}$ with furimazine for $10 \mathrm{~min}$ at $37^{\circ} \mathrm{C}$. Cells were imaged live using a widefield Olympus LV200 Bioluminescence Imaging System as described under Section 2. Images are representative of five independent experiments

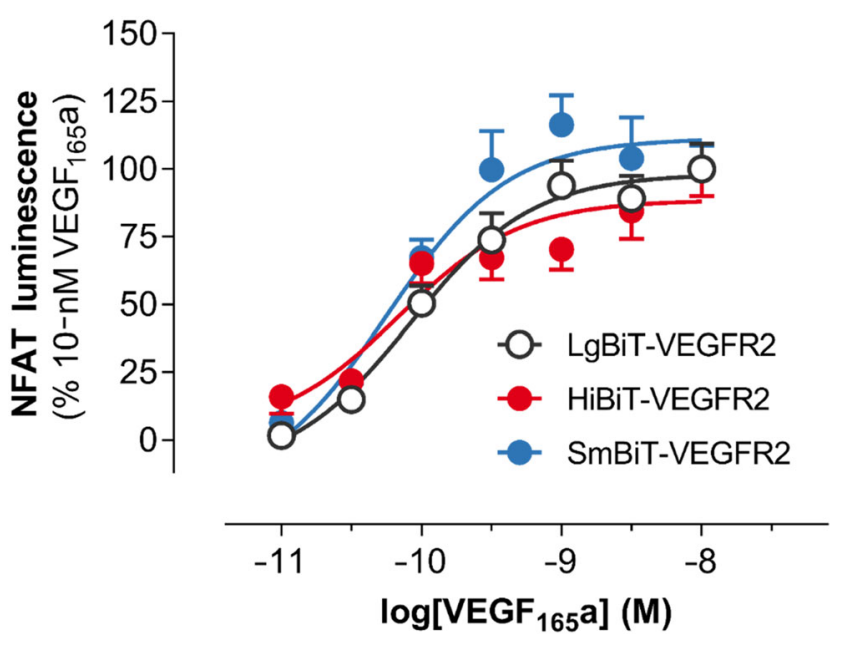

FIGURE 5 Characterisation of NFAT signalling from VEGFR2 tagged with LgBiT, HiBiT, or SmBiT moieties. HEK293T cells stably expressed both NFAT-ReLuc2P and either LgBiT-VEGFR2, HiBiTVEGFR2, or SmBiT-VEGFR2. (a) Cells were serum starved for $24 \mathrm{~h}$. On the day of experimentation, cells were stimulated with increasing concentrations of VEGF 165 for $5 \mathrm{~h}$ and incubated at $37^{\circ} \mathrm{C} / 5 \% \mathrm{CO}_{2}$. Data were normalised to mean vehicle (0\%) or 10-nM unlabelled VEGF $_{165}$ a (100\%) per experiment. Data are expressed as mean \pm SEM from five independent experiments with duplicate wells per experiment

NanoLuc-VEGFR2 alone $\left(7.29 \times 10^{6} \mathrm{~min}^{-1} \cdot \mathrm{M}^{-1}\right.$; Peach, Kilpatrick, et al., 2018). We then directly compared the real-time binding profile for a saturating concentration of $V_{E G F}{ }_{165} b-T M R$ between the NanoBiT complex and cells expressing NanoLuc-tagged receptors alone in matched time course experiments (Figure 7b). Compared to NanoLuc-VEGFR2, the small decline in BRET signal after a peak at 20 min in intact cells was absent when monitored at the NanoBiT complex for VEGF 165 b-TMR. There was no BRET detected between
VEGF $_{165} \mathrm{~b}-\mathrm{TMR}$ and NanoLuc-NRP1. However, this selective ligand had a distinct long-term kinetic profile at the VEGFR2/NRP1 complex compared to VEGFR2 alone (Figure 7b).

Kinetic experiments were repeated with four concentrations of VEGF $_{165}$ a-TMR (Figure 7c). Unlike VEGF 165 b-TMR, there was a small decline in BRET ratio between 30 and 60 min for VEGF 165 a-TMR at the HiBiT complex (Figure 7c). Association binding curves were globally fitted to kinetic data from the initial 20 min due to this decline (Table 1). VEGF $_{165}$ a-TMR had a slower dissociation rate constant $\left(k_{\text {off }}\right)$ at the HiBiT complex (0.046 $\pm 0.007 \mathrm{~min}^{-1}$; Table 1 ) compared to that previously reported for NanoLuc-NRP1 expressed alone $\left(0.26 \mathrm{~min}^{-1}\right.$; Peach, Kilpatrick, et al., 2018). As a consequence, the kinetic binding profile for 10-nM VEGF 165 a-TMR was directly compared between the NanoBiT complex and either NanoLuc-VEGFR2 or NanoLuc-NRP1 (Figure 7d). VEGF $_{165}$ a-TMR association kinetics at the NanoBiT complex in the initial $20 \mathrm{~min}$ were more comparable to NanoLuc-VEGFR2 than NanoLuc-NRP1 (NanoBiT $k_{\text {obs }}=0.33 \pm 0.04 \mathrm{~min}^{-1}$, NanoLuc-VEGFR2 $k_{\text {obs }}=0.31 \pm 0.03 \mathrm{~min}^{-1}, \quad$ NanoLuc-NRP1 $k_{\text {obs }}=0.93 \pm 0.09 \mathrm{~min}^{-1}$; $n=5$ per group). These observed rate constants were significantly slower at the complex than NRP1 alone (repeated-measures ANOVA and Holm-Šidák's multiple comparisons; $P<.05, n=5$ for each). These data suggest that the ligand binding profile for VEGF $_{165} a-T M R$ at the NanoBiT complex reflected VEGFR2 binding kinetics, as opposed to the faster binding observed at NRP1.

\section{6 | Fluorescent VEGF-A kinetics were similar for the SmBiT complex}

Considering the distinct kinetic observations at the HiBiT complex, we further probed ligand binding kinetics at the SmBiT complex to explore possible influences of the NanoBiT tag characteristics (Dixon et al., 2016). Using four concentrations of $V_{E G F}{ }_{165} b-T M R$, binding 
(a)

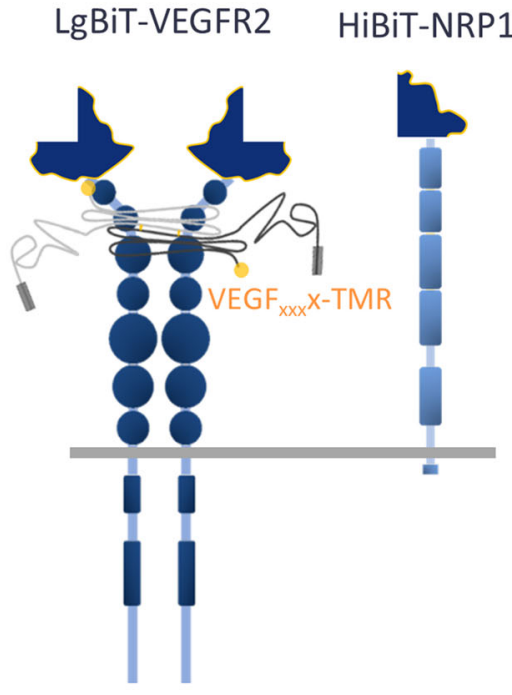

No BRET

(b)

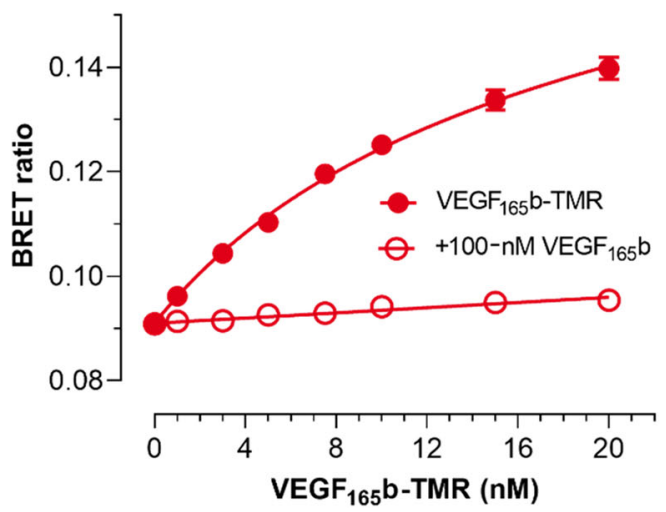

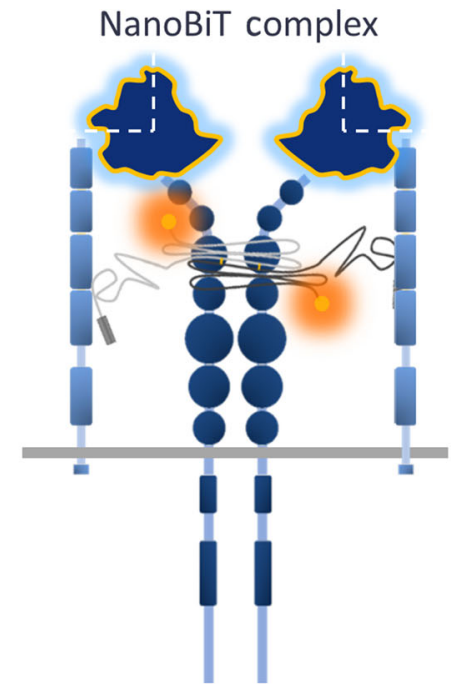

BRET

(c)

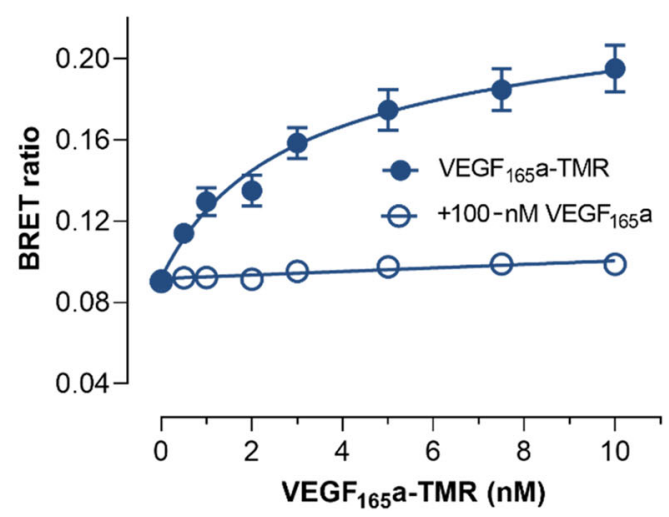

FIGURE 6 Saturation binding of VEGF 165 -TMR and VEGF 165 a-TMR at a HiBiT complex of VEGFR2 and NRP1. (a) Fluorescent VEGF-A ligand binding was monitored at a defined complex of LgBiT-VEGFR2 and HiBiT-NRP1. Two distinct fluorescent VEGF-A isoforms were investigated, denoted by the $x$; the $a$ isoform can engage both VEGFR2 and NRP1 whereas the $b$ isoform cannot interact with NRP1. In the presence of furimazine, individual receptors do not emit luminescence in isolation. Upon NanoBiT complementation, luminescence emissions can excite the tetramethylrhodamine (TMR) in proximity. NanoBiT therefore only acts as a luminescent donor when VEGFR2 and NRP1 are in complex. (b,c) HEK293T cells were transfected in six-well plates with equal amounts of LgBiT-VEGFR2 (750-ng cDNA per well) and HiBiT-NRP1 (750-ng cDNA per well). Following $24 \mathrm{~h}$, transfected cells were seeded in 96-well plates. On the day of experimentation, cells were incubated with increasing concentrations of VEGF 165 -TMR (b) or VEGF 165 a-TMR (c). This was performed in the presence or absence of 100-nM VEGF 165 b (b) or $\mathrm{VEGF}_{165}$ a (c) to determine non-specific binding. This is shown with fluorescent ligand only or with unlabelled ligand. Following $60 \mathrm{~min}$ at $37^{\circ} \mathrm{C}, 10-\mu \mathrm{M}$ furimazine was added for $10 \mathrm{~min}\left(37^{\circ} \mathrm{C}\right)$. Emissions were measured on the PHERAstar platereader. BRET ratios are expressed as mean \pm SEM from three independent experiments with duplicate wells

was monitored over $90 \mathrm{~min}$ (Figure 8a). The binding profile remained elevated throughout the time course with similarities to kinetics observed with the HiBiT complex. Kinetic data were globally fitted to a simple exponential association (Table 1). VEGF 165 b-TMR had a slower dissociation rate $\left(k_{\text {off }}\right)$ from the SmBiT complex compared to the HiBiT complex. Plotting the individual observed association rate constants $\left(k_{\text {obs }}\right)$ against VEGF 165 b-TMR concentration, there was a linear relationship observed at both $\mathrm{HiBiT}$ and SmBiT complexes (Figure 8b). The interaction between $V_{E G F}{ }_{165} \mathrm{~b}-\mathrm{TMR}$ and the NanoBiT complex can therefore be defined as a first-order reaction.
Binding kinetics were also monitored at the SmBiT complex using four concentrations of VEGF 165 a-TMR (Figure 8c). From the fitted data from the initial 20-min period using a global fit, there were no differences between the association kinetic parameters derived for VEGF $_{165}$ a-TMR for the HiBiT and SmBiT complexes. There was a linear relationship between the derived observed association rate $\left(k_{\mathrm{obs}}\right)$ constants and VEGF 165 a-TMR concentration (Figure 8d). Despite having the potential to bind to both receptors within the complex, the interaction between VEGF 165 a-TMR and the NanoBiT complex could also be defined by a first-order reaction. 
(a)

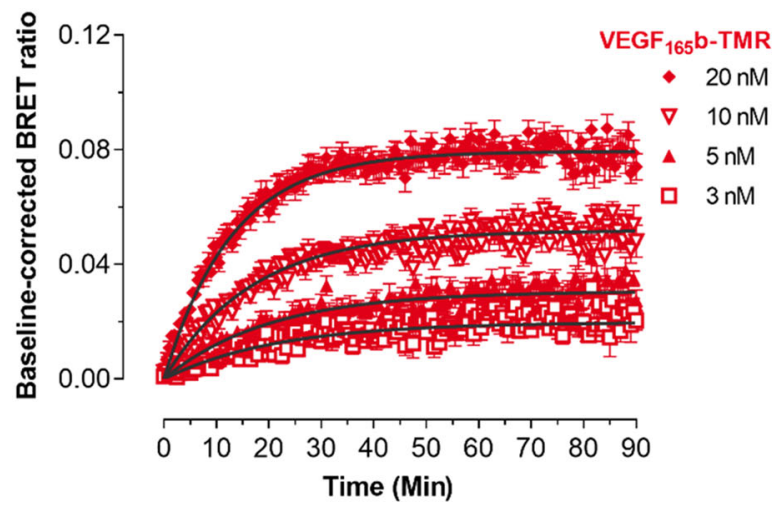

(c)

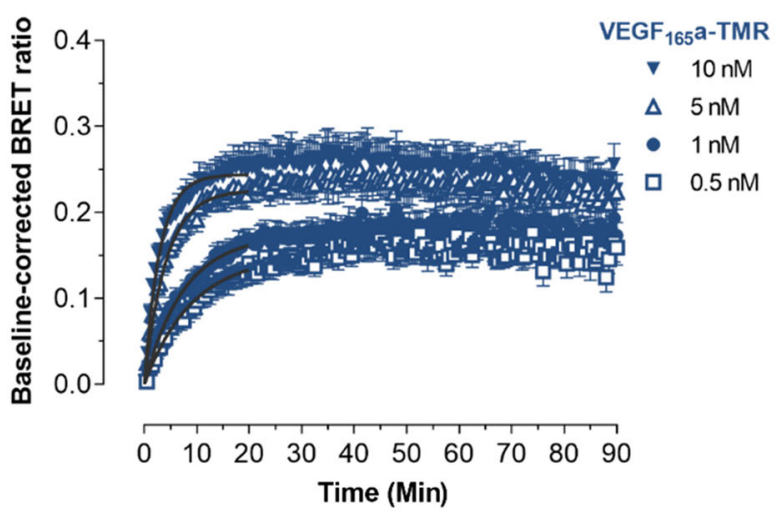

(b)

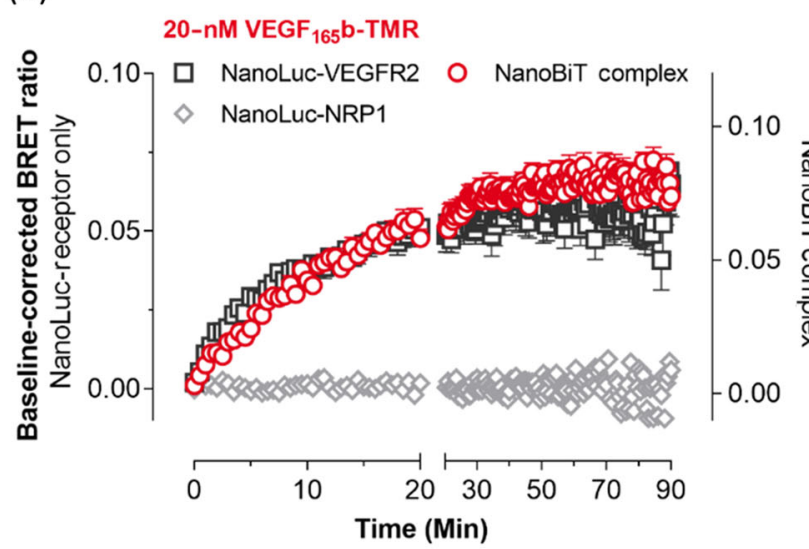

(d)

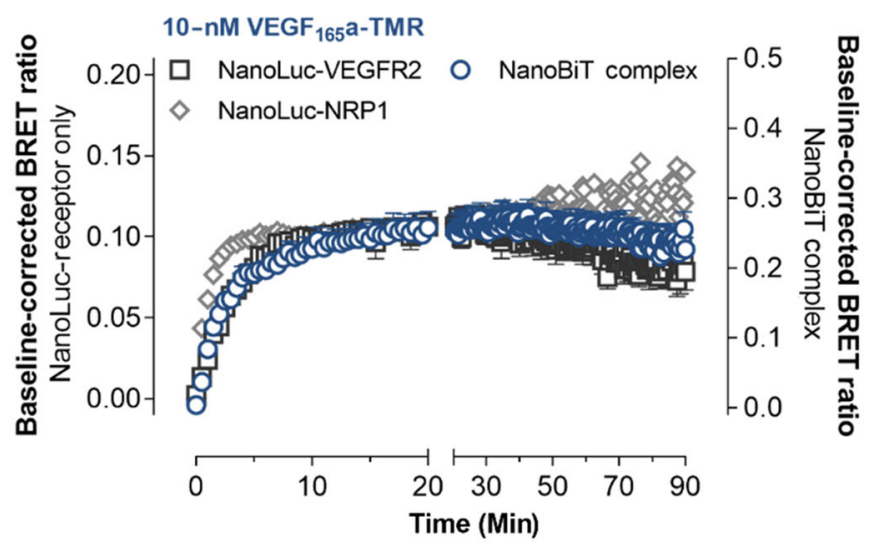

FIGURE 7 Real-time binding of fluorescent VEGF-A isoforms at the NanoBiT complex compared to isolated receptors. (a) HEK293T cells were transfected in six-well plates with equal amounts of LgBiT-VEGFR2 (750-ng cDNA per well) and HiBiT-NRP1 (750-ng cDNA per well). Alternatively, cells were transfected with equal amounts of NanoLuc-VEGFR2 or NanoLuc-NRP1 (750-ng cDNA per well) and empty pcDNA3.1/ Zeo vector (750-ng cDNA per well). Following $24 \mathrm{~h}$, transfected cells were seeded in 96-well plates. On the day of experimentation, cells were pretreated with furimazine $\left(10 \mu \mathrm{M}\right.$ ) and left to equilibrate at $37^{\circ} \mathrm{C}$ for $10 \mathrm{~min}$. (a) Cells expressing the NanoBiT complex (LgBiT-VEGFR2/HiBiTNRP1) were stimulated with four concentrations of VEGF $165 \mathrm{~b}-\mathrm{TMR}$ added at $x=0$. Kinetic data were fitted to a global association model with an unconstrained $k_{\text {on }}$ from the 90-min time course. (b) On the same plate, the real-time binding profile of 20-nM VEGF ${ }_{165} \mathrm{~b}$-TMR was monitored in cells only expressing either NanoLuc-VEGFR2 or NanoLuc-NRP1 (left hand Y-axis). This was directly compared to binding of the same concentration of VEGF $165 \mathrm{~b}$-TMR at the LgBiT-VEGFR2/HiBiT-NRP1 NanoBiT complex (right hand Y-axis). (c) Cells expressing the HiBiT complex were stimulated with four concentrations of $\mathrm{VEGF}_{165} \mathrm{a}-\mathrm{TMR}$. Kinetic data were fitted to a global association model without a constrained $k_{\text {on }}$ from the initial 20 min due to the latter decline in BRET ratio. (d) The real-time binding profile of a saturating concentration of VEGF $165 \mathrm{a}-\mathrm{TMR}$ $(10 \mathrm{nM})$ was compared between cells expressing LgBiT-VEGFR2/HiBiT-NRP1 (right hand Y-axis) to cells only expressing NanoLuc-VEGFR2 or NanoLuc-NRP1 (left hand Y-axis). For each experiment, emissions were simultaneously measured on the PHERAstar FS platereader every $30 \mathrm{~s}$ for $90 \mathrm{~min}$ at $37^{\circ} \mathrm{C}$. BRET ratios were baseline corrected to vehicle at each time point per experimental replicate. In (b) and (d), the $x$ axis was split to highlight the initial association (20 min) and long-term BRET signal (90 min). Data represent mean \pm SEM from five independent experiments with duplicate wells. Derived $k_{o n}, k_{\text {off }}$, and kinetic $p K_{d}$ parameters are in Table 1

\section{7 | Similar complex pharmacology using a binding-dead mutant of NRP1}

In addition to comparing binding between selective and non-selective fluorescent VEGF-A isoforms, site-directed mutagenesis provided an alternative approach to probe the contribution of NRP1 engagement to the pharmacological characteristics of the VEGFR2/NRP1 complex. Using a previously characterised binding-dead NRP1 mutant (Y297A; Herzog et al., 2011; Fantin et al., 2014; Peach, Kilpatrick, et al., 2018), comparisons were made using the same ligand in the absence of interactions between $\mathrm{VEGF}_{165} \mathrm{a}-\mathrm{TMR}$ and NRP1 within the heteromeric NanoBiT complex (Figure 9a). Upon co-expression of LgBiT-VEGFR2 and either HiBiT- or SmBiT-NRP1 (Y297A), there were high luminescence emissions resulting from NanoBiT complementation (Figure 9b). Luminescence emissions from this NanoBiT complex were comparable to wild-type NRP1, implying that this amino acid residue was not required for constitutive VEGFR2/NRP1 complex formation. NanoBiT constructs expressed in isolation from their complementary fragment also had minimal luminescence emissions in the presence of furimazine (Figure 9b). Isolating ligand 
TABLE 1 Summary of binding parameters derived at the NanoBiT complex for VEGF 165 b-TMR and VEGF $_{165} \mathrm{a}-\mathrm{TMR}$, compared to published values from receptors expressed alone

\begin{tabular}{clll} 
& Kinetic $\mathrm{pK}_{\mathbf{d}}$ & $\boldsymbol{k}_{\text {on }}\left(\mathbf{m i n}^{-1} \cdot \mathbf{M}^{-1}\right)$ & $\boldsymbol{k}_{\text {off }}\left(\mathbf{m i n}^{-1}\right)$ \\
\hline VEGF $_{165}$ b-TMR & & & \\
HiBiT complex & $7.81 \pm 0.10(5)$ & $2.29 \times 10^{6} \pm 0.30 \times 10^{6}(5)$ & $0.037 \pm 0.007^{*}(5)$ \\
SmBiT complex & $8.43 \pm 0.17(5)$ & $2.94 \times 10^{6} \pm 0.55 \times 10^{6}(5)$ & $0.012 \pm 0.003(5)$ \\
VEGF $_{165}$ a-TMR & & & \\
HiBiT complex & $8.83 \pm 0.12(5)$ & $3.12 \times 10^{7} \pm 0.43 \times 10^{7}(5)$ & $0.046 \pm 0.007(5)$ \\
SmBiT complex & $8.83 \pm 0.31(5)$ & $2.83 \times 10^{7} \pm 0.69 \times 10^{7}(5)$ & $0.046 \pm 0.020(5)$ \\
\hline
\end{tabular}

Note: The number of independent experiments are shown in parentheses, each with duplicate wells. Kinetic parameters were derived from a global association fit from the full 90-min time course (VEGF 165 b-TMR) or the initial 20 min (VEGF 165 a-TMR). Experiments with LgBiT-VEGFR2 and HiBiT-NRP1 (HiBiT complex) are shown in Figure 7a,c. Kinetic experiments with LgBiT-VEGFR2 and SmBiT-NRP1 (SmBiT complex) are shown in Figure 8a,c.

${ }^{*} P<.05$, significantly different from SmBiT complex; Kruskal-Wallis test. binding from this VEGFR2/NRP1 Y297A complex, VEGF 165 a-TMR exhibited saturable binding at the NanoBiT complex (Figure 9c). This was displaced by a high concentration of unlabelled $\mathrm{VEGF}_{165} \mathrm{a}$, confirming that there was low non-specific binding. Derived equilibrium dissociation constants were in the nanomolar range and similar to the wild-type NanoBiT complex $\left(\mathrm{VEGF}_{165} \mathrm{a}\right.$-TMR/NanoBiT Y297A $K_{d}=1.55 \pm 0.38 ; p K_{d} 8.84 \pm 0.11 ; n=3$ ). Binding kinetics at the mutant NanoBiT complex were then monitored using four concentrations of VEGF 165 a-TMR (Figure 9d). This had a profile identical to that of VEGF 165 a-TMR at the wild-type HiBiT complex (Figure 7c), in that there was a small decline in BRET ratio following $30-60 \mathrm{~min}$. Association kinetics were derived from the initial 20 min using a global fit $\quad\left(k_{\text {on }}=3.71 \times 10^{7} \pm 0.21 \times 10^{7} \mathrm{~min}^{-1} \cdot \mathrm{M}^{-1}\right.$; $k_{\text {off }}=0.054 \pm 0.008 \mathrm{~min}^{-1}$; kinetic $\mathrm{p} K_{\mathrm{d}}=8.85 \pm 0.04 ; n=5$ ). These data suggest that VEGF 165 a-TMR bound the NanoBiT complex with similar kinetics, regardless of the ability to simultaneously engage NRP1.

\section{4 | DISCUSSION}

NanoBiT technologies were used to quantify the real-time binding of two fluorescent VEGF-A isoforms at a defined receptor/co-receptor complex between VEGFR2 and NRP1 in living cells at $37^{\circ} \mathrm{C}$. Previous work identified differences between VEGFR2 and NRP1 pharmacology in terms of their binding kinetics and localisation when expressed on their own (Peach, Kilpatrick, et al., 2018). VEGFR2 and NRP1 are, however, endogenously co-expressed together in endothelial cells and tumour cells (Fantin et al., 2013; Koch et al., 2014; Lee-Montiel et al., 2015; Prahst et al., 2008; Whitaker et al., 2001). We first demonstrated that full-length VEGFR2 and NRP1 constitutively formed a heteromeric complex in living HEK293T cells. To then probe how this specific receptor/co-receptor heteromer interacted with ligand, we established a novel approach to quantify fluorescent VEGF-A binding at a defined complex using split NanoBiT fragments (Dixon et al., 2016). VEGFR2 and NRP1 tagged at their N-terminus with $\mathrm{HiBiT}$ and $\mathrm{LgBiT}$ tags led to NanoBiT complementation with minimal luminescence when each was expressed alone. The formation of this NanoBiT complex could be prevented by increasing amounts of an unlabelled version of one of the heteromer components. As such, the BRET signal was specific to interactions between the VEGFR2/ NRP1 heteromer (BRET donor) and fluorescent VEGF-A (BRET acceptor). This allowed us to monitor ligand binding to a defined RTK/co-receptor oligomeric complex. Uncomplemented VEGFR2 or NRP1 that still binds to the fluorescent ligand does not, however, contribute to the BRET signal due to the lack of complemented donor luminescence and the requirement for donor and acceptor to be within $10 \mathrm{~nm}$ of each other.

Numerous biochemical techniques have suggested that VEGFR2 and NRP1 form heteromeric complexes, including coimmunoprecipitation studies in endothelial cells (Gelfand et al., 2014; Prahst et al., 2008; Whitaker et al., 2001) and proximity ligation assays using antibodies in situ on tumour tissue (Koch et al., 2014). Förster resonance energy transfer (FRET) has also been used to demonstrate complex formation using truncated VEGFR2 and full-length NRP1 tagged with fluorophores at their C-terminus (King et al., 2018). Here, we initially used BRET between full-length VEGFR2 and NRP1 tagged at their $\mathrm{N}$-terminus with NanoLuc or a fluorophore to confirm complex formation in the absence of added VEGF-A. The approach monitored complex formation that originated at the cell membrane, because membrane-impermeant fluorophore-conjugated HaloTag or SnapTag substrates were used. Basal VEGFR2/NRP1 complex formation was also confirmed using both HiBiT-VEGFR2 and LgBiT-NRP1 complementation and the reverse LgBiT-VEGFR2 and HiBiT-NRP1 orientation.

Following the discovery that $\mathrm{VEGF}_{165}$ a had faster binding kinetics for binding to NRP1 than to VEGFR2 when expressed on their own (Peach, Kilpatrick, et al., 2018), it was proposed that the presence of NRP1 might enhance VEGF 165 a binding to the heteromeric complex. The application of both NanoBiT technology and NanoBRET to monitor exclusively VEGF 165 a-TMR binding to VEGFR2/NRP1 complexes allowed us to test this hypothesis directly. Interestingly, the initial association kinetics (during the first $20 \mathrm{~min}$ ) for VEGF 165 a-TMR binding to the VEGFR2/NRP1 heteromeric complex were closer to those observed at NanoLuc-VEGFR2 in isolation than to NanoLuc-NRP1. This was evident from quantification of the observed rate constant 
(a)

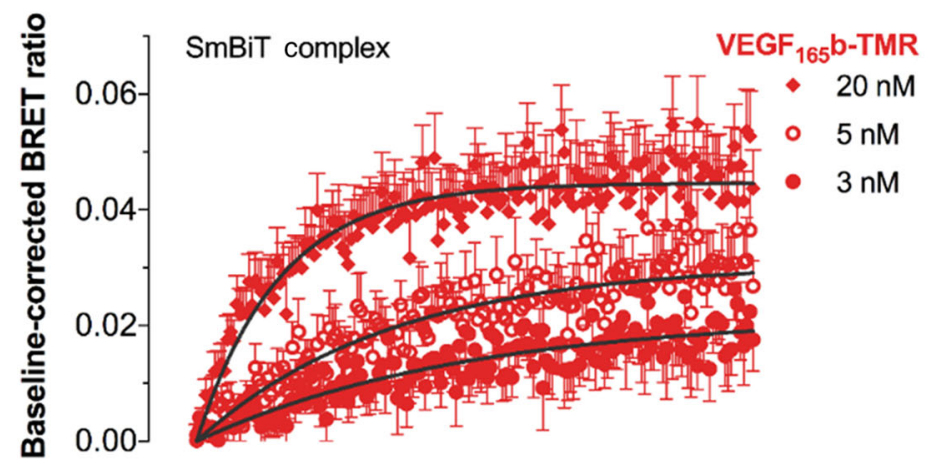

(c)

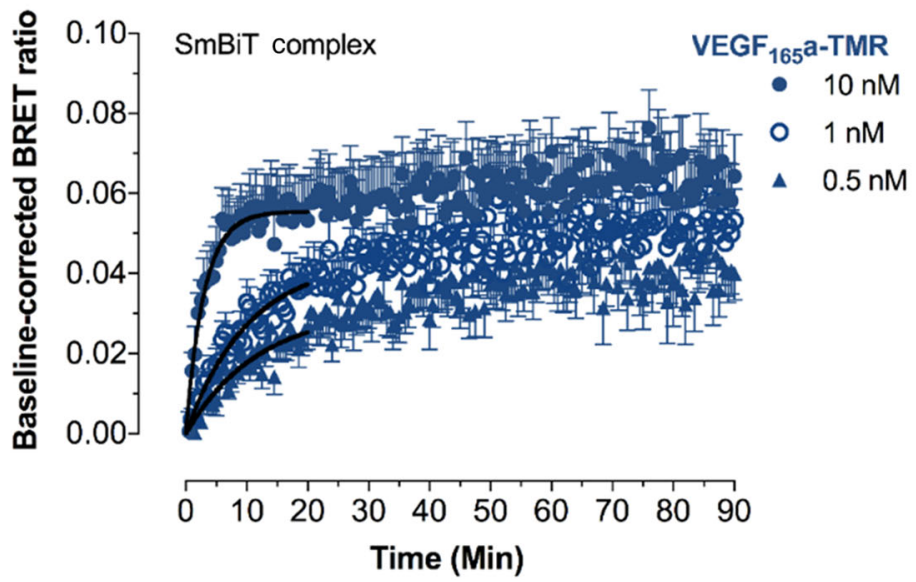

(b)

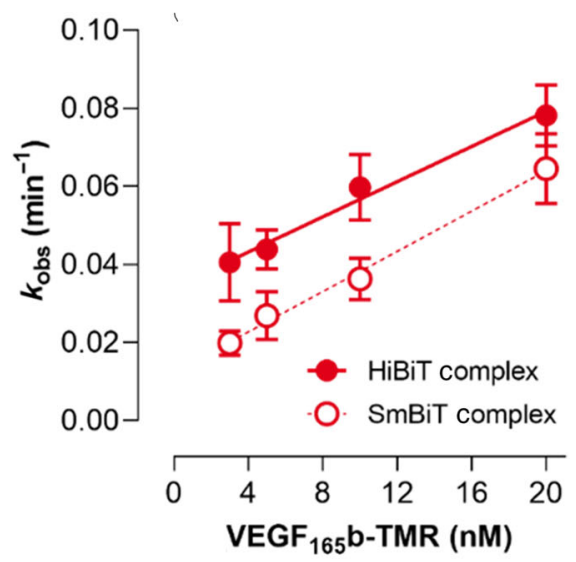

(d)

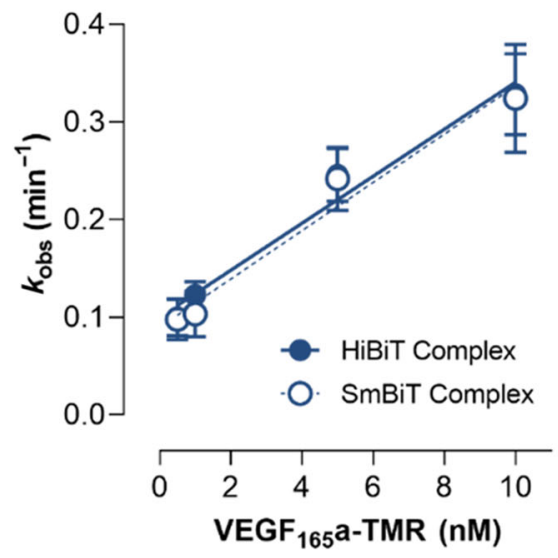

FIGURE 8 Fluorescent VEGF-A binding kinetics at a NanoBiT VEGFR2/NRP1 complex using split tags with lower intrinsic affinity. HEK293T cells were transfected in six-well plates with equal amounts of LgBiT-VEGFR2 (750-ng cDNA per well) and SmBiT-NRP1 (750-ng cDNA per well). Following $24 \mathrm{~h}$, transfected cells were seeded in 96-well plates. Cells were pretreated with furimazine $(10 \mu \mathrm{M})$ and left to equilibrate at $37^{\circ} \mathrm{C}$ for $10 \mathrm{~min}$. (a) Cells were stimulated with four concentrations of VEGF $165 \mathrm{~b}-\mathrm{TMR}$ added at $x=0$. Kinetic data were fitted to a global association model without a constrained $k_{\text {on }}$ from the 90 -min time course. For clarity, the 10-nM data set has not been included in the figure. (b) The derived rate constant, $k_{\mathrm{obs}}$, was obtained from exponential association curves fitted for each of the four fluorescent ligand concentrations. These were plotted against VEGF ${ }_{165} \mathrm{~b}-\mathrm{TMR}$ concentration and fitted against a linear regression (HiBiT complex $y=0.0023 x+0.034, R^{2}=0.46 ; \mathrm{SmBiT}$ complex $y=0.0026 x+0.01, R^{2}=0.65$ ). (c) Cells were stimulated with four concentrations of VEGF $165 a-T M R$. Kinetic data were fitted to a global association model without a constrained $k_{\text {on }}$ from the initial $20 \mathrm{~min}$. For clarity, the 5-nM data set has not been included in the figure. (d) The derived $k_{\text {obs }}$ for each fluorescent ligand at all four concentrations were plotted against each VEGF 165 a-TMR concentration and fit with a linear regression (HiBiT complex $y=0.0024 x+0.10, R^{2}=0.72$; $\mathrm{SmBiT}$ complex $y=0.0025 x+0.09, R^{2}=0.62$ ). Emissions were simultaneously measured on the PHERAstar FS platereader every $30 \mathrm{~s}$ for $90 \mathrm{~min}$ at $37^{\circ} \mathrm{C}$. BRET ratios were baseline corrected to vehicle at each time point per replicate. Data represent mean \pm SEM from five independent experiments with duplicate wells in each independent experiment. Derived $k_{\text {on }}, k_{\text {off }}$, and kinetic $p K_{d}$ parameters are shown in Table 1

from matched experiments at a saturating concentration $(10 \mathrm{nM})$ of fluorescent VEGF 165 a where $k_{\text {obs }}$ was $0.33 \pm 0.04 \mathrm{~min}^{-1}$ for the VEGFR2/NRP1 NanoBiT complex, $0.31 \pm 0.03 \mathrm{~min}^{-1}$ for NanoLucVEGFR2, and $0.93 \pm 0.09 \mathrm{~min}^{-1}$ for NanoLuc-NRP1. Furthermore, the removal of the binding site for VEGF $_{165}$ a on NRP1 by site-directed mutagenesis of residue $\mathrm{Y} 297$ to alanine did not alter the ability of VEGFR2 and NRP1 to form complexes or the binding of VEGF $165^{a-}$ TMR to the heteromeric complex. It is possible therefore that heteromerisation between VEGFR2 and NRP1 masks the high affinity binding site for $\mathrm{VEGF}_{165}$ a on NRP1 and just leaves the VEGFR2 binding site available.

There were some subtle differences between the kinetics of fluorescent VEGF-A isoforms at the VEGFR2/NRP1 heteromeric complex. Pro-angiogenic VEGF 165 and anti-angiogenic $V E_{16} F_{165} \mathrm{~b}$ are functionally distinct VEGF-A isoforms, although these isoforms only differ by six amino acid residues at their C-terminus. Despite observed physiological distinctions between VEGF-A isoforms, there were no differences observed at the level of ligand binding to 
(a)

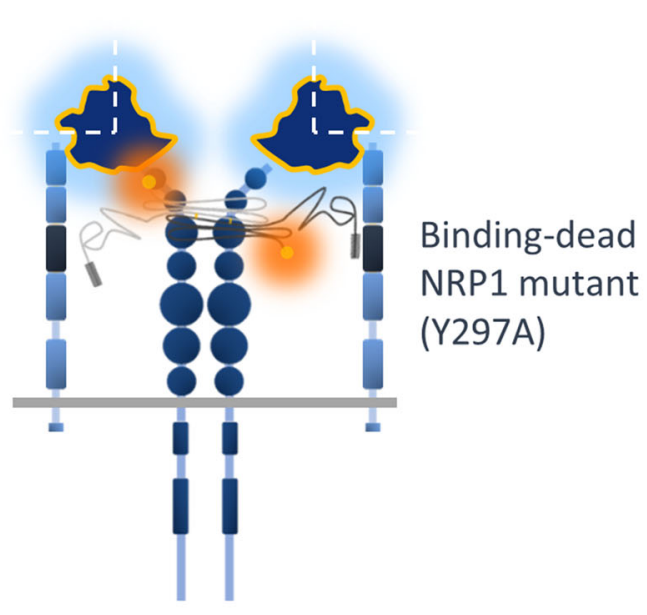

(c)

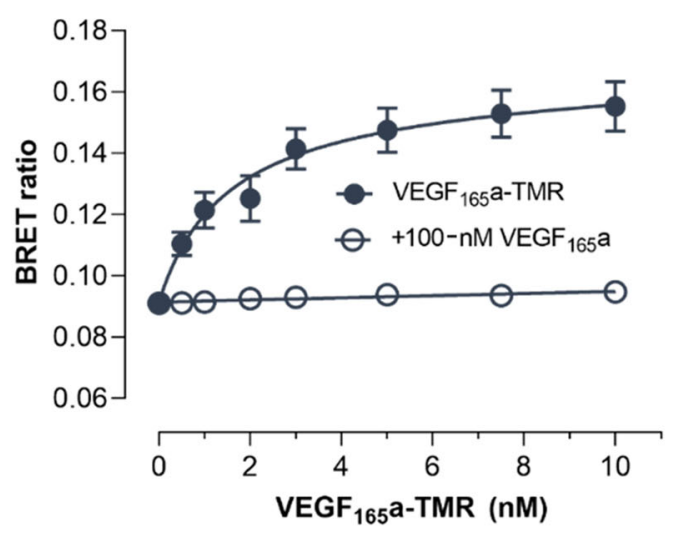

(b)

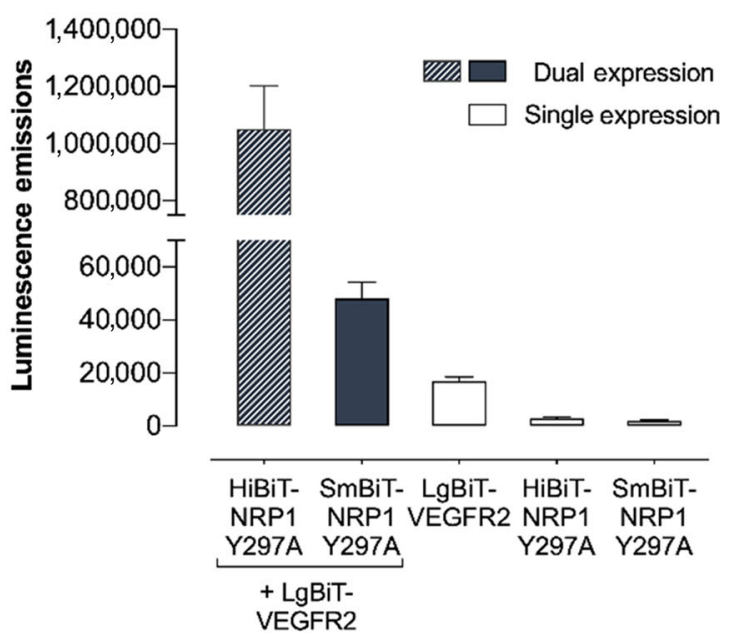

(d)

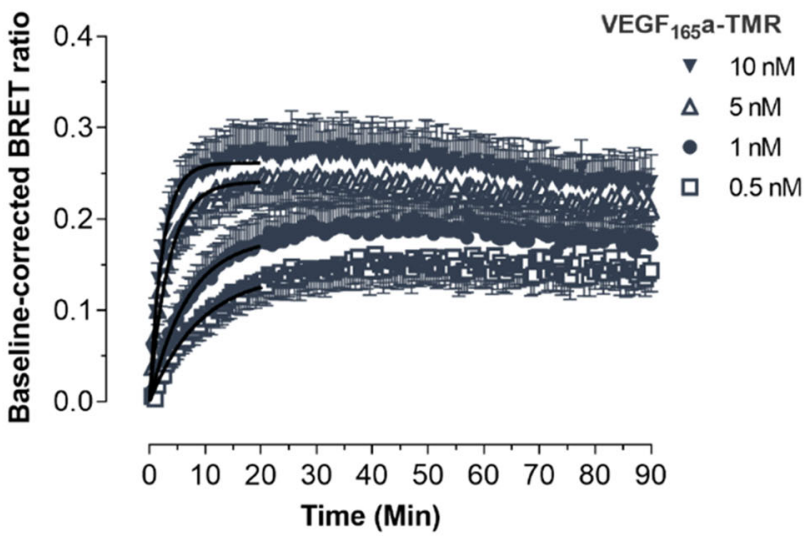

FI G URE 9 Ligand binding of VEGF 165 a-TMR at a NanoBiT complex with a binding-dead NRP1 mutant. (a) VEGF 165 a-TMR ligand binding was monitored at a defined NanoBiT complex between LgBiT-VEGFR2 and a HiBiT-NRP1 VEGF-A binding-dead mutant in the b1 domain (Y297A).

(b) HEK293T cells were transiently transfected in 96-well plates with LgBiT-VEGFR2, HiBiT-NRP1 Y297A, or SmBiT-NRP1 Y297A (50-ng cDNA per well). Dual expression cells expressed a complemented NanoBiT complex with the HiBiT or SmBiT tag. Cells also expressed single constructs (empty bars) were transfected with 50-ng cDNA per well of empty pcDNA3.1/Zeo vector. Cells were incubated with $10-\mu \mathrm{M}$ furimazine in HBSS/0.1\% BSA for $10 \mathrm{~min}\left(37^{\circ} \mathrm{C}\right)$. Luminescence emissions $(475-505 \mathrm{~nm})$ were measured by the PHERAstar FS platereader. Data were normalised to untransfected cells (0\%) and HiBiT-NRP1 Y297A/LgBiT-VEGFR2 (100\%) per experiment. Data are expressed as mean \pm SEM from five independent experiments, each with triplicate wells. (c,d) HEK293T cells were transfected in six-well plates with equal amounts of LgBiTVEGFR2 (750-ng cDNA per well) and HiBiT-NRP1 Y297A (750-ng cDNA per well). Following $24 \mathrm{~h}$, transfected cells were seeded in 96-well plates. (c) On the day of experimentation, cells were incubated with increasing concentrations of VEGF $165^{a-T M R, ~ w i t h ~ o r ~ w i t h o u t ~} 100-n M$ VEGF $_{165}$ a to determine non-specific binding. Following $60 \mathrm{~min}$ at $37^{\circ} \mathrm{C}, 10-\mu \mathrm{M}$ furimazine was added for 10 min $\left(37^{\circ} \mathrm{C}\right)$. Emissions were measured on the PHERAstar platereader (550-LP/460-480 nm). BRET ratios are expressed as mean \pm SEM from three independent experiments with duplicate wells. Derived equilibrium dissociation constants $\left(K_{d}\right)$ are in the text. (d) Cells were pretreated with furimazine $(10 \mu \mathrm{M})$ and left to equilibrate at $37^{\circ} \mathrm{C}$ for $10 \mathrm{~min}$. Cells were incubated with four concentrations of VEGF $165^{2}$-TMR. Kinetic data were fitted to a global association model without a constrained $k_{\text {on }}$ from the initial 20 min. Emissions were simultaneously measured on the PHERAstar FS platereader every $30 \mathrm{~s}$ for $90 \mathrm{~min}$ at $37^{\circ} \mathrm{C}$. BRET ratios were baseline corrected to vehicle at each time point per experimental replicate. Data represent mean $\pm \mathrm{SEM}$ from five independent experiments with duplicate wells. Derived $k_{\text {on }}, k_{\text {off }}$, and kinetic $p K_{d}$ parameters are noted in the text

NanoLuc-VEGFR2 when it was expressed alone (Peach, Kilpatrick, et al., 2018). VEGF $165 \mathrm{~b}$ is, however, selective for VEGFR2 and unable to interact with NRP1 (Peach, Kilpatrick, et al., 2018). The real-time BRET signal for $\mathrm{VEGF}_{165} \mathrm{~b}$-TMR remained elevated in intact cells at the NanoBiT complex over the full 90-min time course. This resembled observations made with NanoLuc-VEGFR2 in membrane preparations and was quite different to the decline in BRET signal normally observed in intact HEK293T cells (Peach et al., 2019). In contrast, the profile for $\mathrm{VEGF}_{165} \mathrm{a}-\mathrm{TMR}$ at the HiBiT complex had a small decrease at latter time points, albeit to a lesser extent than at NanoLuc-VEGFR2 in intact cells (Peach et al., 2019). This reduction in BRET signal for NanoLuc-VEGFR2 following 
20 min has been linked to VEGF-A/VEGFR2 endocytosis leading to a change in localisation and local $\mathrm{pH}$, as this decline was absent in membrane preparations and not observed for binding to NanoLucNRP1 (Peach et al., 2019). These data suggest that the presence of NRP1 in VEGFR2 heteromeric complexes may reduce the extent of VEGFR2 endocytosis normally seen when VEGFR2 is expressed alone.

Imaging studies exploited the compatibility of HaloTag and SnapTag technologies to label distinct receptors co-expressed by the same cell to monitor co-localisation at $37^{\circ} \mathrm{C}$. Unlike immunofluorescent antibody labelling, these experiments can be performed in living cells and do not require cell fixation or cell permeabilisation to access internalised receptors. These distinct tags confirmed that VEGFR2 was largely intracellular whereas NRP1 was highly localised around the plasma membrane when they were both co-expressed in the same cell. NRP1 was also localised in filopodia-like projections in HEK293T cells that resembled the filopodia of endothelial tip cells (Fantin et al., 2013, 2015). Although co-localisation studies were limited by the axial resolution limit of basic confocal microscopy, experiments monitoring receptor-receptor BRET confirmed that VEGFR2 and NRP1 were in proximity $(<10 \mathrm{~nm})$. Live cell confocal imaging and bioluminescence imaging data both suggested that VEGFR2 and NRP1 were co-localised in both intracellular compartments and at the plasma membrane. VEGFR2 is subject to macropinocytosis in the absence or presence of ligand (Basagiannis \& Christoforidis, 2016; Basagiannis et al., 2016). This bulk transport mechanism could therefore non-selectively engulf surrounding NRP1 in living cells. There is evidence in HUVECs for co-localisation between VEGFR2 and NRP1 both at the plasma membrane in the absence of stimulation (LeeMontiel et al., 2015) or within intracellular sites following 20-min VEGF $_{165}$ a stimulation (Muhl et al., 2017). As the NanoLuc/NanoBiT substrate furimazine is membrane permeable, luminescence could be emitted from complexes anywhere in the cell regardless of subcellular localisation.

NanoBiT technologies take advantage of NanoLuc, a small enzyme engineered from a deep sea shrimp with bright, ATPindependent luminescence emissions (Hall et al., 2012). The small, 11-amino-acid NanoBiT fragment also has mutations that confer differing intrinsic affinities for the LgBiT fragment. For example, HiBiT has a much higher intrinsic affinity for LgBiT than SmBiT (Dixon et al., 2016). Luminescence emissions from HiBiT-containing complexes were higher than for the corresponding SmBiT-containing complex, as observed previously for NanoBiT-tagged GPCRs (Botta et al., 2019). This is likely to be due to differences in the affinity of LgBiT for SmBiT or HiBiT. Dixon et al. (2016) confirmed the rapid kinetics of NanoBiT tag complementation (seconds) and, therefore, this is unlikely to affect the ligand binding kinetics monitored at the VEGFR2/NRP1 complex (minutes). The intrinsic affinity between $\mathrm{HiBiT}$ and LgBiT can vary according to the expression system and protein conformation, as observed for chemokine GPCRs using the purified exogenous tag in different assay set-ups (White et al., 2020). While the intrinsic affinity between NanoBiT tags must be taken into consideration, luminescence emissions from both $\mathrm{HiBiT}$ and $\mathrm{SmBiT}$ complexes were displaceable by increasing amounts of competing NRP1 (Figure 3d). The kinetic parameters derived from HiBiT and SmBiT complexes were also comparable, suggesting that VEGFR2NRP1 complex formation was not being driven by the affinity of the $\mathrm{HiBiT}$ tag for LgBiT.

Despite its ability to up-regulate VEGF-A/VEGFR2 signalling in physiological and pathophysiology, the mechanism by which NRP1 up-regulates VEGFR2 signalling remains largely unknown. NRP1 can interact with a number of other growth factors (Banerjee et al., 2006; Rizzolio et al., 2012; West et al., 2005). Therefore, understanding how NRP1 co-expression influences RTK function has implications for other receptors contributing to cancer drug resistance. Our NanoBiT approach allowed us to isolate VEGF-A ligand binding at a defined complex of VEGFR2 and NRP1 and suggested that NRP1 did not increase the affinity or association binding kinetics of $\mathrm{VEGF}_{165}$ a at VEGFR2. While NRP1 appeared to have no direct effect on ligand binding to a VEGFR2/NRP1 complex expressed within the same cell, NRP1 (which is quite often expressed endogenously at higher levels than VEGFR2) could still act as a reservoir for growth factors and create a localised gradient due to its interactions with the extracellular matrix (Shintani et al., 2006; Windwarder et al., 2016).

In summary, we have described here an approach using NanoBiT technology and NanoBRET to monitor in real time the binding of VEGF-A isoforms to defined heteromeric complexes containing both VEGFR2 and NRP1. Understanding the ligand binding properties of a specific heteromeric receptor oligomer is important for studying the molecular pharmacology of individual VEGF-A isoforms in primary cells, such as endothelial and tumour cells, which often endogenously co-express both receptors. This specific technique allowed us to determine for the first time the ligand binding kinetics of VEGF $165 \mathrm{a}^{\mathrm{a}}$ TMR and VEGF 165 b-TMR to a defined VEGFR2-NRP1 complex. We were able to use bioluminescence imaging and confocal microscopy to determine that VEGFR2-NRP1 complexes are localised in both intracellular compartments and at the plasma membrane. At the plasma membrane, the presence of NRP1 within the heteromeric complex appeared to reduce the extent of agonist-induced VEGFR2 endocytosis normally observed when it is expressed alone. The presence of NRP1 within the VEGFR2-NRP1 heteromeric complexes did not enhance VEGF 165 a-TMR binding, and a NRP1 binding-dead mutant (Y297A) had no effect on the binding of VEGF 165 a-TMR, or the formation of VEGFR2-NRP1 complexes, suggesting that the high affinity binding site for $V_{E G F}$ 165 a NRP1 might be masked within the heteromeric complexes. In keeping with this conclusion, $\mathrm{VEGF}_{165} \mathrm{~b}-\mathrm{TMR}$, which does not bind to NRP1, had a very similar binding profile to the heteromeric complex to that observed with VEGF $_{165}$ a-TMR. This approach to monitor the binding profile of defined oligomeric complexes should be applicable to a wide range of receptor systems and facilitate drug discovery aimed a heteromeric complex. This approach could also be developed further to observe ligand interactions with a specified oligomer in vivo due to the small size of the $\mathrm{HiBiT} / \mathrm{SmBiT}$ tag and bright luminescence emissions, in a similar way to that we have recently reported for a GPCR tagged with the full-length NanoLuc (Alcobia et al., 2018). Given the clinical 
importance of therapeutic agents targeting VEGF or VEGFR2 in cancer and other pathologies, understanding the mechanism by which NRP1 up-regulates VEGF-A/VEGFR2 signalling is a priority for identifying new targets to improve the long-term efficacy and adverse effects of VEGF-targeted therapeutics in cancer.

\section{ACKNOWLEDGEMENTS}

This work was funded by Biotechnology and Biological Sciences Research Council (BBSRC) (grant number BB/L019418/1), the Medical Research Council (grant number MR/N020081/1), and the British Pharmacological Society. C.J.P. held an AJ Clark studentship from the British Pharmacological Society. L.E.K. holds an Anne McLaren Fellowship from the University of Nottingham. We thank the Centre of Membrane Proteins and Receptors (COMPARE) for support and Promega Corporation for synthesising VEGF 165 a-TMR and VEGF VE5 $_{165} \mathrm{~b}$ TMR and providing HaloTag- and NanoLuc-tagged constructs. We also thank the School of Life Sciences Imaging (SLIM) team for support in imaging facilities and analysis.

\section{AUTHOR CONTRIBUTIONS}

S.J.H., J.W., L.E.K., and C.J.P. conceived the study. C.J.P., L.E.K., S.J.H., and J.W. participated in research design. C.J.P. and L.E.K. conducted experiments. C.J.P. and S.J.H. performed data analysis. C.J.P., L.E.K., J.W., and S.J.H. wrote or contributed to the writing of the manuscript.

\section{CONFLICT OF INTEREST}

The authors declare no conflicts of interest.

\section{DECLARATION OF TRANSPARENCY AND SCIENTIFIC RIGOUR}

This Declaration acknowledges that this paper adheres to the principles for transparent reporting and scientific rigour of preclinical research as stated in the BJP guidelines for Design \& Analysis, and as recommended by funding agencies, publishers, and other organisations engaged with supporting research.

\section{DATA AVAILABILITY STATEMENT}

The data that support the findings of this study are available from the corresponding authors upon reasonable request.

\section{ORCID}

Chloe J. Peach (D) https://orcid.org/0000-0001-6315-1170

Laura E. Kilpatrick (iD https://orcid.org/0000-0001-6331-5606 Jeanette Woolard (D) https://orcid.org/0000-0001-5406-6847 Stephen J. Hill (D) https://orcid.org/0000-0002-4424-239X

\section{REFERENCES}

Alcobia, D. C., Ziegler, A. I., Kondrashov, A., Comeo, E., Mistry, S., Kellam, B., Chang, A., Woolard, J., Hill, S. J., \& Sloan, E. K. (2018). Visualising ligand-binding to a GPCR in vivo using nanoBRET. iscience, 6, 280-288.

Alexander, S. P. H., Fabbro, D., Kelly, E., Mathie, A., Peters, J. A., Veale, E. L., Armstrong, J. F., Faccenda, E., Harding, S. D., Pawson, A. J., Sharman, J. L., Southan, C., Davies, J. A., \& CGTP
Collaborators. (2019). THE CONCISE GUIDE TO PHARMACOLOGY 2019/20: Catalytic receptors. British Journal of Pharmacology, 176, S247-S296. https://doi.org/10.1111/bph.14751

Banerjee, S., Sengupta, K., Dhar, K., Mehta, S., D'Amore, P., Dhar, G., \& Banerjee, S. K. (2006). Breast cancer cells secreted platelet-derived growth factor-induced motility of vascular smooth muscle cells is mediated through neuropilin-1. Molecular Carcinogenesis, 45, 871-880. https://doi.org/10.1002/mc.20248

Basagiannis, D., \& Christoforidis, S. (2016). Constitutive endocytosis of VEGFR2 protects the receptor against shedding. The Journal of Biological Chemistry, 291, 16892-16903. https://doi.org/10.1074/jbc.M116. 730309

Basagiannis, D., Zografou, S., Murphy, C., Fotsis, T., Morbidelli, L., Ziche, M., Bleck, C., Mercer, J., \& Christoforidis, S. (2016). VEGF induces signalling and angiogenesis by directing VEGFR2 internalisation via macropinocytosis. Journal of Cell Science, 129, 4091-4104. https://doi.org/10.1242/jcs.188219

Botta, J., Bibic, L., Killoran, P., McCormick, P., \& Howell, L. (2019). Design and development of stapled transmembrane peptides that disrupt the activity of G-protein coupled receptor oligomers. The Journal of Biological Chemistry, 294, 16587-16603. https://doi.org/10.1074/jbc. RA119.009160

Brozzo, M. S., Bjelic, S., Kisko, K., Schleier, T., Leppánen, V.-M., Alitalo, K., Winkler, F. K., \& Ballmer-Hofer, K. (2011). Thermodynamic and structural description of allosterically regulated VEGF receptor 2 dimerization. Blood, 119, 1781-1788.

Carmeliet, P. (2005). Angiogenesis in life, disease and medicine. Nature, 438, 932-936. https://doi.org/10.1038/nature04478

Cébe Suarez, S., Pieren, M., Cariolato, L., Arn, S., Hoffman, U., Bogucki, A., Manlius, C., Wood, J., \& Ballmer-Hofer, K. (2006). A VEGF-A splice variant defective for heparan sulfate and neuropilin-1 binding shows attenuated signaling through VEGFR-2. Cellular and Molecular Life Sciences, 63, 2067-2077. https://doi.org/10.1007/s00018-006-6254-9

Chung, A. S., \& Ferrara, N. (2011). Developmental and pathological angiogenesis. Annual Review of Cell and Developmental Biology, 27, 563-584. https://doi.org/10.1146/annurev-cellbio-092910-154002

Delcombel, R., Janssen, L., Vassy, R., Gammons, M., Haddad, O., Richard, B., Letourneur, D., Bates, D., Hendricks, C., Waltenberger, J., Starzec, A., Sounni, N. E., Noël, A., Deroanne, C., Lambert, C., \& Colige, A. (2013). New prospects in the roles of the C-terminal domains of VEGF-A and their cooperation for ligand binding, cellular signaling and vessels formation. Angiogenesis, 16, 353-371. https:// doi.org/10.1007/s10456-012-9320-y

Dixon, A. S., Schwinn, M. K., Hall, M. P., Zimmerman, K., Otto, P., Lubben, T. H., Butler, B. L., Binkowski, B. F., Machleidt, T., Kirkland, T. A., Wood, M. G., Eggers, C. T., Encell, L. P., \& Wood, K. V. (2016). NanoLuc complementation reporter optimized for accurate measurement of protein interactions in cells. ACS Chemical Biology, 11, 400-408. https://doi.org/10.1021/acschembio.5b00753

Djordjevic, S., \& Driscoll, P. C. (2013). Targeting VEGF signalling via the neuropilin co-receptor. Drug Discovery Today, 18, 447-445. https:// doi.org/10.1016/j.drudis.2012.11.013

Eswarappa, S. M., Potdar, A. A., Koch, W. J., Fan, Y., Vasu, K., Lindner, D., Willard, B., Graham, L. M., DiCorleto, P. E., \& Fox, P. L. (2014). Programmed translational readthrough generates antiangiogenic VEGF-Ax. Cell, 157, 1605-1618. https://doi.org/10.1016/j.cell.2014. 04.033

Ewan, L. C., Jopling, H. M., Jia, H., Mittar, S., Bagherzadeh, A., Howell, G. J., Walker, J. H., Zachary, I. C., \& Ponnambalam, S. (2006). Intrinsic tyrosine kinase activity is required for vascular endothelial growth factor receptor 2 ubiquitination, sorting and degradation in endothelial cells. Traffic, 7, 1270-1282. https://doi.org/10.1111/j. 1600-0854.2006.00462.x

Fantin, A., Herzog, B., Mahmoud, M., Yamaji, M., Plein, A., Denti, L., Ruhrberg, C., \& Zachary, I. (2014). Neuropilin 1 (NRP1) hypomorphism 
combined with defective VEGF-A binding reveals novel roles for NRP1 in developmental and pathological angiogenesis. Development, 141, 556-562. https://doi.org/10.1242/dev.103028

Fantin, A., Lampropoulou, A., Gestri, G., Raimondi, C., Senatore, V., Zachary, l., \& Ruhrberg, C. (2015). NRP1 regulates CDC42 activation to promote Filopodia formation in endothelial tip cells. Cell Reports, 11, 1577-1590. https://doi.org/10.1016/j.celrep.2015.05.018

Fantin, A., Schwarz, Q., Davidson, K., Normando, E. M., Denti, L., \& Ruhrberg, C. (2011). The cytoplasmic domain of neuropilin 1 is dispensable for angiogenesis, but promotes the spatial separation of retinal arteries and veins. Development, 138, 4185-4191. https://doi. org/10.1242/dev.070037

Fantin, A., Vieira, J. M., Plein, A., Denti, L., Fruttiger, M., Pollard, J. W., \& Ruhrberg, C. (2013). NRP1 acts cell autonomously in endothelium to promote tip cell function during sprouting angiogenesis. Blood, 121, 2352-2362. https://doi.org/10.1182/blood-2012-05-424713

Gelfand, M. V., Hagan, N., Tata, A., Oh, W.-J., Lacoste, B., Kang, K.-T., Kopycinska, J., Bischoff, J., Wang, J. H., \& Gu, C. (2014). Neuropilin-1 functions as a VEGFR2 co-receptor to guide developmental angiogenesis independent of ligand binding. eLife, 3, e03720. https://doi.org/ 10.7554/eLife.03720

Goel, H. L., \& Mercurio, A. M. (2013). VEGF targets the tumour cell. Nature Reviews. Cancer, 13, 871-882. https://doi.org/10.1038/ $\operatorname{nrc3627}$

Hall, M. P., Unch, J., Binkowski, B. F., Valley, M. P., Butler, B. L., Wood, M. G., Otto, P., Zimmerman, K., Vidugiris, G., Machleidt, T., Robers, M. B., Benink, H. A., Eggers, C. T., Slater, M. R., Meisenheimer, P. L., Klaubert, D. H., Fan, F., Encell, L. P., \& Wood, K. V. (2012). Engineered luciferase reporter from a deep sea shrimp utilizing a novel imidazopyrazinone substrate. ACS Chemical Biology, 7, 1848-1857. https://doi.org/10.1021/cb3002478

Herzog, B., Pellet-Many, C., Britton, G., Hartzoulakis, B., \& Zachary, I. C. (2011). VEGF binding to NRP1 is essential for VEGF stimulation of endothelial cell migration, complex formation between NRP1 and VEGFR2, and signaling via FAK Tyr407 phosphorylation. Molecular Biology of the Cell, 22, 2766-2776. https://doi.org/10.1091/mbc.e0912-1061

Jopling, H. M., Howell, G. J., Gamper, N., Ponnambalam, S. (2011). The VEGFR2 receptor tyrosine kinase undergoes constitutive endosometo-plasma membrane recycling. Biochemical and Biophysical Research Communications, 410(2), 170-176. https://doi.org/10.1016/j.bbrc. 2011.04.093.

Jopling, H. M., Odell, A. F., Hooper, N. M., Zachary, I. C., Walker, J. H., Ponnambalam, S. (2009). Rab GTPase regulation of VEGFR2 trafficking and signaling in endothelial cells. Arteriosclerosis, Thrombosis, and Vascular Biology, 29(7), 1119-1124. https://doi.org/10.1161/atvbaha. 109.186239.

Jubb, A. M., Strickland, L. A., Liu, S. D., Mak, J., Schmidt, M., \& Koeppen, H. (2012). Neuropilin-1 expression in cancer and development. The Journal of Pathology, 226, 50-60. https://doi.org/10.1002/ path.2989

Kawamura, H., Li, X., Harper, S. J., Bates, D., \& Claesson-Welsh, L. (2008). Vascular endothelial growth factor (VEGF)-A165b is a weak in vitro agonist for VEGF receptor-2 due to lack of coreceptor binding and deficient regulation of kinase activity. Cancer Research, 68, 4683-4692. https://doi.org/10.1158/0008-5472.CAN-07-6577

Kilpatrick, L. E., Friedman-Ohana, R., Alcobia, D. C., Riching, K., Peach, C. J., Wheal, A., Briddon, S. J., Robers, M. B., Zimmerman, K., Machleidt, T., \& Wood, K. V. (2017). Real-time analysis of the binding of fluorescent VEGF 165 a to VEGFR2 in living cells: Effect of receptor tyrosine kinase inhibitors and fate of internalized agonist-receptor complexes. Biochemical Pharmacology, 136, 62-75. https://doi.org/10. 1016/j.bcp.2017.04.006

King, C., Wirth, D., Workman, S., \& Hristova, K. (2018). Interactions between NRP1 and VEGFR2 molecules in the plasma membrane.
Biochimica et Biophysica Acta, Biomembranes, 1860, 2118-2125. https://doi.org/10.1016/j.bbamem.2018.03.023

Koch, S., Tugues, S., Li, X., Gualandi, L., \& Claesson-Welsh, L. (2011). Signal transduction by vascular endothelial growth factor receptors. The Biochemical Journal, 437, 169-183. https://doi.org/10.1042/ BJ20110301

Koch, S., van Meeteren, L. A., Morin, E., Testini, C., Weström, S., Björkelund, H., Le Jan, S., Adler, J., Berger, P., \& Claesson-Welsh, L. (2014). NRP1 presented in trans to the endothelium arrests VEGFR2 endocytosis, preventing angiogenic signaling and tumor initiation. Developmental Cell, 28, 633-646. https://doi.org/10.1016/j.devcel. 2014.02.010

Lee, S. W., Lee, J. E., Yoo, C. Y., Ko, M. S., Park, C. S., \& Yang, S. H. (2014) NRP-1 expression is strongly associated with the progression of pituitary adenomas. Oncology Reports, 32, 1537-1542. https://doi.org/10. 3892/or.2014.3392

Lee-Montiel, F. T., Li, P., \& Imoukhuede, P. I. (2015). Quantum dot multiplexing for the profiling of cellular receptors. Nanoscale, 7, 18504-18514. https://doi.org/10.1039/C5NR01455G

Leppanen, V. M., Prota, A. E., Jeltsch, M., Anisimov, A., Kalkkinen, N., Strandin, T., Lankinen, H., Goldman, A., Ballmer-Hofer, K., \& Alitalo, K. (2010). Structural determinants of growth factor binding and specificity by VEGF receptor 2. Proceedings. National Academy of Sciences. United States of America, 107, 2425-2430. https://doi.org/10.1073/ pnas.0914318107

Mamluk, R., Gechtman, Z., Kutcher, M. E., Gasiunas, N., Gallagher, J., \& Klagsbrun, M. (2002). Neuropilin-1 binds vascular endothelial growth factor 165, placenta growth factor-2, and heparin via its b1b2 domain. The Journal of Biological Chemistry, 277, 24818-24825. https://doi. org/10.1074/jbc.M200730200

Muhl, L., Folestad, E. B., Gladh, H., Wang, Y., Moessinger, C., Jakobsson, L., \& Eriksson, U. (2017). Neuropilin 1 binds plateletderived growth factor (PDGF)-D and is a co-receptor in PDGFD/PDGF receptor $\beta$ signaling. Journal of Cell Science, 130, 1365-1378. https://doi.org/10.1242/jcs.200493

Parker, M. W., Xu, P., Li, X., \& Vander Kooi, C. W. (2012). Structural basis for selective vascular endothelial growth factor-A (VEGF-A) binding to neuropilin-1. The Journal of Biological Chemistry, 287, 11082-11089. https://doi.org/10.1074/jbc.M111.331140

Peach, C. J., Kilpatrick, L. E., Friedman-Ohana, R., Zimmerman, K., Robers, M. B., Wood, K. V., Woolard, J., \& Hill, S. J. (2018). Real-time ligand binding of fluorescent VEGF-A isoforms that discriminate between VEGFR2 and NRP1 in living cells. Cell Chemical Biology, 25, 1208-1218. https://doi.org/10.1016/j.chembiol.2018.06.012

Peach, C. J., Kilpatrick, L. E., Woolard, J., \& Hill, S. J. (2019). Comparison of the ligand binding properties of fluorescent VEGF-A isoforms to VEGFR2 in living cells and membrane preparations using NanoBRET. British Journal of Pharmacology, 176, 3220-3235. https://doi.org/10. 1111/bph.14755

Peach, C. J., Mignone, V. W., Arruda, M. A., Hill, S. J., Kilpatrick, L. E., \& Woolard, J. (2018). Molecular pharmacology of VEGF-A isoforms: Binding and signalling at VEGFR2. International Journal of Molecular Sciences, 19, 1-27.

Prahst, C., Héroult, M., Lanahan, A. A., Uziel, N., Kessler, O., Shraga-Heled, N., Simons, M., Neufeld, G., \& Augustin, H. G. (2008). Neuropilin-1-VEGFR-2 complexing requires the PDZ-binding domain of neuropilin-1. The Journal of Biological Chemistry, 283, 25110-25114. https://doi.org/10.1074/jbc.C800137200

Rizzolio, S., Rabinowicz, N., Rainero, E., Lanzetti, L., Serini, G., Norman, J., Neufeld, G., \& Tamagnone, L. (2012). Neuropilin-1-dependent regulation of EGF-receptor signaling. Cancer Research, 72, 5801-5811. https://doi.org/10.1158/0008-5472.CAN-12-0995

Roy, S., Bag, A. K., Singh, R. K., Talmadge, J. E., Batra, S. K., \& Datta, K. (2017). Multifaceted role of neuropilins in the immune system: Potential targets for immunotherapy. Frontiers in Immunology, 8, 1-27. 
Ruch, C., Skiniotis, G., Steinmetz, M. O., Walz, T., \& Ballmer-Hofer, K. (2007). Structure of a VEGF-VEGF receptor complex determined by electron microscopy. Nature Structural \& Molecular Biology, 14, 249-250. https://doi.org/10.1038/nsmb1202

Shintani, Y., Takashima, S., Asano, Y., Kato, H., Liao, Y., Yamazaki, S., Tsukamoto, O., Seguchi, O., Yamamoto, H., Fukushima, T., Sugahara, K., Kitakaze, M., \& Hori, M. (2006). Glycosaminoglycan modification of neuropilin-1 modulates VEGFR2 signaling. The EMBO Journal, 25, 3045-3055. https://doi.org/10.1038/sj.emboj.7601188

Simons, M., Gordon, E., \& Claesson-Welsh, L. (2016). Mechanisms and regulation of endothelial VEGF receptor signalling. Nature Reviews. Molecular Cell Biology, 17, 611-625. https://doi.org/10.1038/nrm. 2016.87

Soker, S., Miao, H. Q., Nomi, M., Takashima, S., \& Klagsbrun, M. (2002). VEGF165 mediates formation of complexes containing VEGFR-2 and neuropilin-1 that enhance VEGF165-receptor binding. Journal of Cellular Biochemistry, 85, 357-368. https://doi.org/10.1002/jcb. 10140

Soker, S., Takashima, S., Miao, H. Q., Neufeld, G., \& Klagsbrun, M. (1998). Neuropilin-1 is expressed by endothelial and tumor cells as an isoform-specific receptor for vascular endothelial growth factor. Cell, 92, 735-745. https://doi.org/10.1016/S0092-8674(00)81402-6

Stoddart, L., Johnstone, E. K. M., Wheal, A. J., Goulding, J., Robers, M. B., Machleidt, T., Wood, K. V., Hill, S. J., \& Pfleger, K. D. (2015). Application of BRET to monitor ligand binding to GPCRs. Nature Methods, 12, 661-663. https://doi.org/10.1038/nmeth.3398

Stoddart, L. A., Kilpatrick, L. E., \& Hill, S. J. (2017). NanoBRET approaches to study ligand binding to GPCRs and RTKs. Trends in Pharmacological Sciences, 39, 136-147.

Vander Kooi, C. W., Jusino, M. A., Perman, B., Neau, D. B., Bellamy, H. D., \& Leahy, D. J. (2007). Structural basis for ligand and heparin binding to neuropilin B domains. Proceedings. National Academy of Sciences. United States of America, 104, 6152-6157. https://doi.org/10.1073/pnas.0700043104

Vempati, P., Popel, A. S., \& Mac Gabhann, F. (2014). Extracellular regulation of VEGF: Isoforms, proteolysis, and vascular patterning. Cytokine \& Growth Factor Reviews, 25, 1-19. https://doi.org/10.1016/j.cytogfr. 2013.11.002

West, D. C., Rees, C. G., Duchesne, L., Patey, S. J., Terry, C. J., Turnbull, J. E., Delehedde, M., Heegaard, C. W., Allain, F., Vanpouille, C., Ron, D., \& Fernig, D. G. (2005). Interactions of multiple heparin binding growth factors with neuropilin-1 and potentiation of the activity of fibroblast growth factor-2. The Journal of Biological Chemistry, 280, 13457-13464. https://doi.org/10.1074/jbc. M410924200

Whitaker, G. B., Limberg, B. J., \& Rosenbaum, J. S. (2001). Vascular endothelial growth factor receptor-2 and neuropilin-1 form a receptor complex that is responsible for the differential signaling potency of VEGF $_{165}$ and VEGF $_{121}$. The Journal of Biological Chemistry, 276, 25520-25531. https://doi.org/10.1074/jbc.M102315200

White, C. W., Caspar, B., Vanyai, H. K., Pfleger, K. D. G., \& Hill, S. J. (2020). CRISPR-mediated protein tagging with NanoLuciferase to investigate native chemokine receptor function and conformational changes. Cell Chemical Biology, 27, 499-510. https://doi.org/10.1016/j.chembiol. 2020.01.010

Windwarder, M., Yelland, T., Djordjevic, S., \& Altmann, F. (2016). Detailed characterization of the O-linked glycosylation of the neuropilin-1 c/MAM-domain. Glycoconjugate Journal, 33, 387-397. https://doi.org/ 10.1007/s10719-015-9602-x

Witmer, A. N., Dai, J., Weich, H. A., Vrensen, G. F. J. M., \& Schlingemann, R. O. (2002). Expression of vascular endothelial growth factor receptors 1, 2, and 3 in quiescent endothelia. The Journal of Histochemistry and Cytochemistry, 50, 767-777. https://doi.org/10. 1177/002215540205000603

Woolard, J., Bevan, H. S., Harper, S. J., \& Bates, D. (2009). Molecular diversity of VEGF-A as a regulator of its biological activity. Microcirculation, 16, 572-592. https://doi.org/10.1080/10739680902997333

Woolard, J., Wang, W., Bevan, H. S., Qiu, Y., Morbidelli, L., Pritchardjones, R. O., Cui, T. G., Sugiono, M., Waine, E., Perrin, R., \& Foster, R. (2004). VEGF 165 b, an inhibitory vascular endothelial growth factor splice variant: Mechanism of action, in vivo effect on angiogenesis and endogenous protein expression. Cancer Research, 64, 7822-7835. https://doi.org/10.1158/0008-5472.CAN-04-0934

How to cite this article: Peach CJ, Kilpatrick LE, Woolard J, Hill SJ. Use of NanoBiT and NanoBRET to monitor fluorescent VEGF-A binding kinetics to VEGFR2/NRP1 heteromeric complexes in living cells. Br J Pharmacol. 2021;1-19. https:// doi.org/10.1111/bph.15426 